\title{
Quantitatively modelling kinetics through a visual analysis of the derivative thermogravimetric curves: Application to biomass pyrolysis
}

\author{
Teresa Martí-Rosselló, Jun Li, Leo Lue \\ Department of Chemical and Process Engineering, University of Strathclyde, James Weir Building, 75 Montrose Street, Glasgow G1 1XJ, United \\ Kingdom
}

\begin{abstract}
Analytic relations are developed that directly link visually observable features of differential thermogravimetric (DTG) curves (e.g., peak temperature, height, width, skewness and conversion at the peak) to the parameters of chemical reaction kinetics models (e.g., activation energy and prefactor), which can be used to study the thermal decomposition of solid fuels. General expressions suitable for any reaction model are provided, as well as explicit expressions for $n$th order reactions with a rate constant given by the Arrhenius equation. This approach is illustrated for the pyrolysis of biomass, where it is found to provide a rapid and accurate estimate of the relative contributions of cellulose, hemicellulose, and lignin to the volatile yield, as well as their kinetic parameters. The method offers a simple way to obtain the model reaction kinetics parameters from thermogravimetric data and saves computing time by providing sensible initial values and bounds of the fit parameters.

Keywords: biomass pyrolysis, reaction kinetics, peak shape, deconvolution model
\end{abstract}

\section{Introduction}

Biomass is a promising renewable resource that can be thermally converted directly to heat or to other useful products like bio-oil, gas and char. Biomass pyrolysis consists of heating the biomass in the absence of oxygen, in order to obtain a range of valuable products that can be used to produce clean biofuels and biochemicals. This process is considered to be carbon neutral, and biomass, if sustainably harvested, is a readily available resource. These aspects make products derived from biomass an attractive alternative to fossil fuels, of which there is a depleting supply, and their burning contributes to global warming.

Biomass can be characterized in terms of its chemical, elemental and proximate analysis. Common values for the proximate analysis of lignocellulosic biomass are 5-10\% moisture, less than 5\% of ash, 10-20\% fixed carbon, and

\footnotetext{
${ }^{*}$ Corresponding author

Email addresses: teresa.marti@strath.ac.uk (Teresa Martí-Rosselló ), jun.li@strath.ac.uk (Jun Li ), leo.lue@strath.ac.uk (Leo Lue )
} 
$70-80 \%$ of volatile content [1]. The main chemical components of biomass are hemicellulose, cellulose, and lignin; their relative proportions vary across different types of biomass. They each undergo different thermal transformations due to their specific chemical structure, and, therefore, their degradation occurs in distinct temperature ranges. Hemicellulose is a branched polymer, usually represented by the molecule xylan $\left(\mathrm{C}_{5} \mathrm{H}_{8} \mathrm{O}_{4}\right)$, and its decomposition takes place between 200 and $300^{\circ} \mathrm{C}$. Cellulose is an unbranched polymer of glucose $\left(\mathrm{C}_{6} \mathrm{H}_{10} \mathrm{O}_{5}\right)$ that decomposes between 240 and $350^{\circ} \mathrm{C}$. Lignin is an amorphous and complex polymer, decomposing between 250 and $500^{\circ} \mathrm{C}$ [6, 7]. It is ackowledged that interactions between the components can affect their decomposition [2, 3, 4], but this is typically assumed not to be significant [5].

Due to the heterogeneity of biomass and that its decomposition might comprise parallel and consecutive reactions, only approximate reaction mechanisms can be drawn. To use it as an alternative to fossil fuels, research on the kinetics of its thermochemical processes is needed to achieve high energy efficiency [8]. Research on biomass pyrolysis is currently focused on both fundamental and practical aspects of the process, including intrinsic reaction kinetics and heat and mass transfer at the particle and reactor scales, which are the main controlling processes to take into account for reactor and process design, mainly focused on product yields distribution and prediction. Typically, at the early stage of biomass pyrolysis, condensable (bio-oil) and non-condensable (gas) volatiles are released from the solid biomass, then, if the condensable volatiles are not removed from the reaction zone, they can further react to form more permanent gases [9].

The main operating conditions in biomass pyrolysis that affect the product yields are the heating rate, the maximum temperature at which the biomass is held, and the residence time of the volatile in the reaction zone [10]. Depending on the desired product, these parameters are adjustable to produce preferred end-products. In order to increase the liquid yield, a high heating rate to a moderate temperature of about $500^{\circ} \mathrm{C}$ and a short residence time of the volatile, in the order of seconds, that are subsequently quenched into bio-oil are required. The operating conditions may also affect the composition of the bio-oil recovered from the condensable volatiles. Furthermore, smaller particle sizes of feedstock in pyrolysis process also contribute to an increase in liquid yield [11, 12]. Kinetic analysis is often used to elucidate the reaction mechanisms of biomass decomposition. A good reaction mechanism should be able to predict biomass decomposition for a wide range of operating conditions and biomass types, in order to be coupled with transport models and thus, scale up the process for industrial purposes.

Thermogravimetric analysis (TGA) is a widely used standard approach to study the thermal decomposition of biomass. In TGA, a sample is subjected to a temperature program, and changes in the mass of the sample are recorded. The differential thermogravimetric (DTG) curve describes the mass loss rate of the sample with temperature or time. A DTG curve from biomass pyrolysis typically features several peaks, each of them corresponding to the individual 
decomposition of each chemical component.

In analyzing a biomass sample from a single thermogravimetric run and treating the biomass as a homogeneous material, the results cannot be generalized to other types of biomass or alternative process conditions.

A practical approach to increasing the applicability of an experiment to a wider range of biomass types is to assume that the overall decomposition of a biomass sample can be described as a sum of the individual decomposition of its main chemical components and assuming that there is no significant interactions between them [5].

A multi-component kinetic mechanism, comprises the individual decomposition behavior of each component, and each reaction scheme can comprise one or more consecutive reactions, the power-law reaction model being the most used. Detailed kinetic mechanisms often take into account intermediate reactions and product yields, for instance, kinetic schemes with an intermediate step and schemes that comprise numerous consecutive reactions and predict specific species in each product phase, like the ones by Ranzi et al. [13, 14].

As for the applicability of the kinetic parameters to a wider range of operating conditions, a possible solution is to evaluate simultaneously thermogravimetric data from different TGA/DTG curves, obtaining one or more shared kinetic parameters. With multi-component mechanisms, simultaneous evaluation of TGA/DTG curves becomes more complex than dealing with a single component, and it can be carried out in several ways. For example, Gronli et al. simultaneously fitted DTG curves from different types of biomass using the same heating rate, and determined a single activation energy for all samples but different pre-exponential factors and component fractions [15]. In contrast, Branca et al. used DTG data from the same type of biomass at different heating rates, and determined a single set of kinetic parameters but different initial component fractions depending on the heating rate [16].

Wet chemistry techniques are generally employed for the determination of biomass composition, although it is also known to be costly and time consuming, and the fractions may be altered in the process. Near infrared (NIR) spectroscopy has been recently reported as an alternative approach to determine biomass composition, however, this technique requires sophisticated equipment and can result in overlapping absorption peaks, making the identification of components more difficult [17]. Deconvolution (or fitting) of TGA data from biomass pyrolysis is another approach that has been studied to obtain the biomass composition [18, 19, 20, 21]. In this case the calculated composition accounts for the volatile fractions of each component in biomass, having biomass over $80 \%$ of volatile matter on a dry, ash-free basis [22]. The biomass composition, found through deconvolution of a DTG curve, is suitable to use in combination with a multi-component kinetic mechanism, because the mechanism describes how the volatile fraction of the biomass is released from the solid.

To distinguish between the individual component reactions within a DTG curve, the different characteristic reaction peaks need to be deconvolved. A deconvolution based on a physical reaction kinetics model normally requires 
an optimization algorithm and an initial estimate of the model parameters; in addition, the relative contribution of the biomass components is needed if a multi-component mechanism is used. If the model is complex or has numerous adjustable parameters, for many algorithms the initial estimates of the parameters should not be too different from their optimal values; therefore, a good initial estimate of the parameters is paramount to achieve a meaningful fit. These initial values would normally be estimated using the results from previous fits or from literature data, when available. When a good initial estimate of the parameters is not available, evolutionary optimization algorithms, like differential evolution, genetic algorithms [23] or particle swarm optimization [24] can be used. Evolutionary algorithms do not require good starting values to find a global optimum and are typically initialized randomly; however, these optimization algorithms can be very time-consuming.

Efforts have been made to relate the basic features of a DTG curve directly to parameters of reaction kinetics models. Previous work has studied how changes in the order of reaction, activation energy, pre-exponential factor and heating rate affect the shape of the mass loss and DTG curves [25]. The peak temperature is a parameter in the Van-Krevelen approximation for the exponential integral of the Arrhenius equation [26], and it is also central in the widely used model-free Kissinger method, able to extract kinetic parameters from DTG data at different heating rates [27].

A method to calculate the kinetic parameters from a single DTG curve was developed by Kim et al., first using the peak temperature and the height of peak [28], later improved by adding a third shape parameter, which is the conversion at the peak temperature, to determine the reaction order assuming an $n$ th-order reaction mechanism [29]. The effect on the DTG curves of different reaction mechanisms was analyzed and described by Dollimore et al. in terms of their width at half-height and conversion at the peak temperature to obtain a correlation between the shape of the DTG curve and kinetic mechanism [30].

In this work, we develop a method to estimate the parameters of a reaction kinetics model directly from the features of a DTG curve, in particular, the peak temperature, height, width, skewness, as well as the conversion at the peak temperature. The method saves computing time by reducing the number of iterations needed to fit experimental DTG data and by providing sensible initial values and constraints to model parameters, independently of the optimization method used and without the need of previous kinetic information on the process. Previous work in the literature that relates the shape of the DTG curve with reaction kinetics relies on numerical analysis. The approach of this paper is entirely analytical and provides a direct link between shape and reaction kinetics. Furthermore, it can be used with any reaction model, such as a distributed activation energy model, as long as the number of model parameters does not exceed the number of measurable shape parameters. In this case, a high number of shape parameters are included in the method and can be used in any combination, although the expressions become increasingly complex. In addition, 
it also allows a simple manner to screen reaction models to determine their applicability.

The remainder of this article is organized as follows. In the next section, the basic description of reaction kinetics is quickly reviewed. Then, in Sec. 3, we present a simple approach to quantitatively characterize the shape of DTG curves, and develop expressions that allow the calculation of the parameters of general reaction kinetic models. Explicit expressions are given for single-step $n$ th-order reactions with rate constants given by the Arrhenius equation. Some examples of the application of these expressions to experimental thermogravimetric data from the literature for the pyrolysis of several types of biomass are given in Sec. 4 to help explain and exemplify the method. Finally, the main findings and future directions of this work are summarized in Sec. 5.

\section{Theory}

\subsection{Kinetics of biomass pyrolysis}

Thermogravimetry has been widely used for the analysis of solid-state kinetics. From the first derivative of the mass loss over time or temperature, the DTG curve is obtained. The DTG curve renders the rate of reaction, which increases with temperature until it reaches a maximum, corresponding to the peak temperature. Figure 1a is an example of experimental data obtained from thermogravimetric analysis, featuring a mass loss and a DTG curve, which could be fitted to a model to determine the kinetic parameters and the chemical composition. From the DTG curve, rather than with the TGA curve, it is easier to distinguish the different reactions corresponding to the different peaks, despite the certain degree of overlapping. Observing the DTG curve in Figure 1a, there are some features from a number of the peaks (i.e. peak temperature, width and height) that can be identified and easily estimated, the rest of features can still be quantitatively approximated using the same units as in the axis of the DTG plot, so a reasonable value could be given within the observed bounds. Most available kinetic models in the literature use a rate law that follows the Arrhenius expression of temperature dependence. The Arrhenius equation has been adapted from homogeneous reactions in gas/liquid phase to solid-state kinetics, and in the latter, it is observed that, for a given reaction, instead of a single value of activation energy for the whole range of temperatures, the value of the activation energy might vary as the reaction proceeds or depending on the experimental conditions [31]. In this regard, various approaches have been explored, like employing different reaction models, distributed activation energy methods (DAEM), iso-conversional methods and simultaneous fitting of thermogravimetric data at different heating rates. When dealing with multi-component and/or multi-step mechanisms with overlapping reactions, simultaneous non-linear fitting of data from different heating programs is advisable [32].

The decomposition of biomass is often expressed as a function of conversion $\alpha=\left(m_{0}-m_{t}\right) /\left(m_{0}-m_{f}\right)$, where $m_{0}$ is the sample initial weight, $m_{t}$ is the weight at a time $t$, and $m_{f}$ refers to the remaining mass when the process has 
finished. The rate law of biomass pyrolysis is assumed to have the form

$$
\frac{d \alpha}{d t}=k(T) f(\alpha),
$$

where $d \alpha / d t$ is the reaction rate, $f(\alpha)$ represents the reaction model, $T$ is the biomass temperature, and $k(T)$ is the rate constant. In decomposition reactions that occur in a kinetically controlled regime, it is reasonable to assume that the temperature of the biomass sample is uniform and equal to the temperature of the environment. Several reaction models may be used [35], for a first order reaction model, the rate of reaction depends only on the temperature and the amount of remaining unreacted sample $(1-\alpha)$.

TG analysis is usually performed with small samples of up to $20 \mathrm{mg}$ and a particle size no larger than $100 \mu \mathrm{m}$ [5]. In the TG analyzer, the temperature of the reaction environment can be set constant in order to perform isothermal analysis or set into a dynamic mode, usually consisting of a constant heating rate of up to $100 \mathrm{~K} \mathrm{~min}^{-1}$ [36]. For non-isothermal analysis at a constant heating rate, the temperature varies with time according to

$$
T=\beta t+T_{0}
$$

where $\beta$ is the heating rate, and $T_{0}$ is the temperature of the biomass at the beginning of the process, usually at room temperature. The change of mass with respect to temperature is given by

$$
\frac{d \alpha}{d T}=\frac{1}{\beta} k(T) f(\alpha) .
$$

For non-isothermal TGA experiments we need to fit the experimental data to a model in order to get the kinetic parameters, the fitting method is based on the least squares minimization of the difference between the experimental and calculated data. To calculate the reaction rate, we integrate Eq. (3):

$$
g(\alpha)=\frac{A}{\beta} \bar{p}(T)
$$

where $A$ is a temperature independent parameter in the expression for the rate constant, and the functions $g(\alpha)$ and $\bar{p}(T)$ are defined as

$$
g(\alpha)=\int_{0}^{\alpha} \frac{d \alpha^{\prime}}{f\left(\alpha^{\prime}\right)}
$$


and

$$
\bar{p}(T)=\frac{1}{A} \int_{T_{0}}^{T} d T^{\prime} k\left(T^{\prime}\right)
$$

In general, the integral $\bar{p}(T)$ cannot be evaluated analytically and must be approximated or determined numerically.

In this section, the Arrhenius equation and its integration has been reviewed in order to help introduce the analytical relations between the DTG peak shape and kinetics, presented in the following section.

\section{Linking peak shape and reaction kinetics}

Key features that characterize the shape of a peak in a DTG curve are the location of the peak $T_{p}$, the height of the peak $H_{p}$, the width from the center to the left of the peak $W_{-}$, and from the center to the right of the peak $W_{+}$at a fraction $\theta$ of its height (see Figure 1b). Given these quantitative measures, the peak can be accurately represented mathematically by the exponential-Gaussian hybrid (EGH) model [37], which is defined as

$$
\alpha_{E G H}^{\prime}(T)=\left\{\begin{array}{ll}
H_{p} \exp \left(-\frac{\left(T-T_{p}\right)^{2}}{2 \sigma^{2}+\tau\left(T-T_{p}\right)}\right) & \text { if } 2 \sigma^{2}+\tau\left(T-T_{p}\right)>0 \\
0 & \text { if } 2 \sigma^{2}+\tau\left(T-T_{p}\right) \leq 0
\end{array} .\right.
$$

This model was originally developed to deconvolve the wide variety of peak shapes that occur in chromatography experiments. The parameters $\sigma$ and $\tau$ are related to the widths $W_{-}$and $W_{+}$as

$$
\begin{aligned}
\sigma^{2} & =-\frac{W_{+} W_{-}}{2 \ln \theta} \\
\tau & =-\frac{W_{+}-W_{-}}{\ln \theta} .
\end{aligned}
$$

For a symmetric peak, $W_{+}=W_{-}, \tau=0$, and the EGH model reduces to a Gaussian peak model; in this case, the full width at half maximum (FWHM) is equal to $2(2 \ln 2)^{1 / 2} \sigma \approx 2.35 \sigma$, where $\sigma$ would be the equivalent to the standard deviation of a Gaussian distribution with the same curvature at the peak. In the EGH model, the value of $\sigma$ depends on the chosen height fraction of the peak, according to Eq. (8). When $W_{-}$is larger than $\mathrm{W}_{+}$, the curve is skewed to the left, and $\tau$ is negative.

In order to relate the characteristics of the peak shape to the parameters of a reaction kinetics model, we perform 
a Taylor series expansion of the natural logarithm of the reaction rate $\ln \alpha^{\prime}(T)$ around the peak temperature $T_{p}$

$$
\ln \alpha^{\prime}(T) \approx\left[\ln \alpha^{\prime}\left(T_{p}\right)\right]+\frac{1}{2}\left[\ln \alpha^{\prime}\left(T_{p}\right)\right]^{\prime \prime}\left(T-T_{p}\right)^{2}+\frac{1}{3 !}\left[\ln \alpha^{\prime}\left(T_{p}\right)\right]^{\prime \prime \prime}\left(T-T_{p}\right)^{3}+\cdots,
$$

and compare this to the corresponding Taylor series expansion of the exponential-Gaussian hybrid $\ln \alpha_{E G H}^{\prime}(T)$

$$
\ln \alpha_{E G H}^{\prime}(T) \approx \ln H_{p}-\frac{\left(T-T_{p}\right)^{2}}{2 \sigma^{2}}+\frac{\tau}{4 \sigma^{4}}\left(T-T_{p}\right)^{3}+\cdots
$$

Matching the expressions order by order, we obtain a set of relationships between the derivatives of the reaction rate at the peak temperature and the parameters that characterize the shape of the peak:

$$
\begin{aligned}
\ln \alpha^{\prime}\left(T_{p}\right) & =\ln H_{p} \\
{\left[\ln \alpha^{\prime}\left(T_{p}\right)\right]^{\prime} } & =0 \\
{\left[\ln \alpha^{\prime}\left(T_{p}\right)\right]^{\prime \prime} } & =-\sigma^{-2} \\
{\left[\ln \alpha^{\prime}\left(T_{p}\right)\right]^{\prime \prime \prime} } & =\frac{3}{2} \frac{\tau}{\sigma^{4}} .
\end{aligned}
$$

The conversion at the peak temperature can be determined from the relation

$$
g\left(\alpha_{p}\right)=\frac{A}{\beta} \bar{p}\left(T_{p}\right) .
$$

Equations (12)-(16) provide the required constraints on the parameters of the reaction kinetics that need to be satisfied in order to reproduce the peak height $H_{p}$, the peak temperature $T_{p}$, the width of the peak $\sigma$, the peak skewness $\tau$, and peak conversion $\alpha_{p}$, respectively.

Figure 2 compares the Gaussian, EGH, and $n$-order reaction kinetics models for the same peak temperatures and same values of $\sigma$ to show the difference between the skewness of the Gaussian and the Arrhenius curves, displaying the latter an asymmetric peak, and how good the EGH model can reproduce both models. The skewness to the left of the Arrhenius DTG curve is common in non-isothermal reactions where the reaction rate increases with time until the unreacted mass is too small to keep increasing and drops, and a skewness to the right would be characteristic of isothermal conditions [38]. 
The derivatives in Eq. (10) can be calculated for a given choice of rate constant $k(T)$ and reaction model $f(\alpha)$,

$$
\begin{aligned}
\ln \alpha^{\prime}\left(T_{p}\right) & =\ln k\left(T_{p}\right)+\ln f\left(\alpha_{p}\right) \\
{\left[\ln \alpha^{\prime}\left(T_{p}\right)\right]^{\prime} } & =\left[\ln k\left(T_{p}\right)\right]^{\prime}+\left[\ln f\left(\alpha_{p}\right)\right]^{\prime} \alpha^{\prime}\left(T_{p}\right) \\
{\left[\ln \alpha^{\prime}\left(T_{p}\right)\right]^{\prime \prime} } & =\left[\ln k\left(T_{p}\right)\right]^{\prime \prime}+\left[\ln f\left(\alpha_{p}\right)\right]^{\prime \prime}\left[\alpha^{\prime}\left(T_{p}\right)\right]^{2} \\
{\left[\ln \alpha^{\prime}\left(T_{p}\right)\right]^{\prime \prime \prime} } & =\left[\ln k\left(T_{p}\right)\right]^{\prime \prime \prime}+\sigma^{-2}\left[\ln k\left(T_{p}\right)\right]^{\prime}+\left[\ln f\left(\alpha_{p}\right)\right]^{\prime \prime \prime}\left[\alpha^{\prime}\left(T_{p}\right)\right]^{3} .
\end{aligned}
$$

\section{1. nth-order reaction model}

In this section, we focus our attention to reaction models of the general form

$$
f(\alpha)=(1-\alpha)^{n},
$$

where $n$ is the order of the reaction. The integral of the $n$th order reaction model is

$$
g(\alpha)=\left[(1-\alpha)^{1-n}-1\right] /(n-1)
$$

Using this in Eq. (4) gives the conversion as an explicit function of the temperature

$$
\alpha=1-\left[1+(n-1) \frac{A}{\beta} \bar{p}(T)\right]^{-1 /(n-1)}
$$

The corresponding expressions for the derivatives of the rate of reaction assuming an $n$ th-order reaction model are

$$
\begin{aligned}
\ln \alpha^{\prime}\left(T_{p}\right) & =\left[\ln \bar{k}\left(T_{p}\right)\right]^{\prime}-\frac{n}{n-1} \ln \left(1+(n-1) \frac{A}{\beta} \bar{p}\left(T_{p}\right)\right)=\ln H \\
{\left[\ln \alpha^{\prime}\left(T_{p}\right)\right]^{\prime} } & =\left[\ln \bar{k}\left(T_{p}\right)\right]^{\prime}-n \frac{A}{\beta} \bar{k}\left(T_{p}\right)\left[1+(n-1) \frac{A}{\beta} \bar{p}\left(T_{p}\right)\right]^{-1}=0 \\
{\left[\ln \alpha^{\prime}\left(T_{p}\right)\right]^{\prime \prime} } & =\left[\ln \bar{k}\left(T_{p}\right)\right]^{\prime \prime}-\frac{1}{n}\left\{\left[\ln \bar{k}\left(T_{p}\right)\right]^{\prime}\right\}^{2}=-\sigma^{-2} \\
{\left[\ln \alpha^{\prime}\left(T_{p}\right)\right]^{\prime \prime \prime} } & =\left[\ln \bar{k}\left(T_{p}\right)\right]^{\prime \prime \prime}-\left[\ln \bar{k}\left(T_{p}\right)\right]^{\prime \prime}\left[\ln \bar{k}\left(T_{p}\right)\right]^{\prime}+\frac{n-2}{n^{2}}\left\{\left[\ln \bar{k}\left(T_{p}\right)\right]^{\prime}\right\}^{3}=\frac{3}{2} \frac{\tau}{\sigma^{4}} \\
\alpha_{p} & =1-\left[1+(n-1) \frac{A}{\beta} \bar{p}\left(T_{p}\right)\right]^{-1 /(n-1)}
\end{aligned}
$$

Equation (24) is related to the height of the peak, Eq. (25) is related to the position of the peak, Eqs. (26) and (27) 
can be used in Eqs. (14) and (15) to obtain expressions for $\sigma$ and $\tau$. Equation (28) gives the conversion at $T_{p}$, which might be useful to identify the best reaction model or, in the case of $n$ th-order reaction models, the order of reaction [39].

The expressions derived up to this point, are general expressions that could be applied to any rate constant for an $n$ th-order reaction model. Below we specialize to the case where the rate constant is given by the Arrhenius equation.

\subsubsection{Arrhenius rate constant}

One common expression for the rate constant $k(T)$ is the Arrhenius equation

$$
k(T)=A e^{-E /(R T)}
$$

where $A$ is the pre-exponential factor, $E$ is the activation energy, and $R$ is the universal gas constant. The corresponding derivatives are

$$
\begin{aligned}
\ln \bar{k}(T) & =-\frac{T_{E}}{T} \\
{[\ln \bar{k}(T)]^{\prime} } & =\frac{T_{E}}{T^{2}} \\
{[\ln \bar{k}(T)]^{\prime \prime} } & =-2 \frac{T_{E}}{T^{3}} \\
{[\ln \bar{k}(T)]^{\prime \prime \prime} } & =6 \frac{T_{E}}{T^{4}} .
\end{aligned}
$$

where $T_{E}=E / R$.

The resulting expressions for the peak width and peak asymmetry parameters for $n$ th-order reactions are

$$
\begin{aligned}
\sigma & =n^{1 / 2} \frac{T_{p}^{2}}{T_{E}}\left(1+2 n \frac{T_{p}}{T_{E}}\right)^{-\frac{1}{2}} \\
\tau & =\frac{2}{3}(n-2) \frac{T_{p}^{2}}{T_{E}}\left[1+2 n\left(\frac{T_{p}}{T_{E}}\right)\right]^{-2}\left[1+\frac{2 n^{2}}{n-2}\left(\frac{T_{p}}{T_{E}}\right)+\frac{6 n^{2}}{n-2}\left(\frac{T_{p}}{T_{E}}\right)^{2}\right]
\end{aligned}
$$

It is interesting to note that the peak width and asymmetry for this reaction kinetics model are dictated by the activation energy and the order of the reaction; they are independent of the pre-exponential factor of the Arrhenius equation. The $T_{p}$ ranges for hemicellulose, cellulose and lignin will be located within the decomposition ranges already given in Sec. 1, and accordingly to the given ranges of typical activation energies of biomass pyrolysis [40], $T_{E}$ would vary for hemicellulose between 9622 and $13952 \mathrm{~K}$, cellulose between 2345 and $34400 \mathrm{~K}$, and lignin between 2165 and 
From knowledge of $E$ and $n$, the pre-exponential factor can be determined from Eq. (25), which leads to

$$
A=\beta \frac{1}{n}\left(\frac{T_{E}}{T_{p}^{2}}\right) e^{T_{E} / T_{p}}\left[1-\frac{(n-1)}{n}\left(\frac{T_{E}}{T_{p}^{2}}\right) e^{T_{E} / T_{p}} \bar{p}\left(T_{p}\right)\right]^{-1}
$$

From this expression it can also be observed that the pre-exponential factor carries the effect of the heating rate.

The height of the DTG peak can be determined from Eq. (24)

$$
H=\frac{A}{\beta} e^{-T_{E} / T_{p}}\left[1+(n-1) \frac{A}{\beta} \bar{p}\left(T_{p}\right)\right]^{-n /(n-1)} .
$$

Figure 3 shows how good the curve characteristics of the EGH model translate into the Arrhenius $n$ th-order model for a range of given values of $\sigma$ and $\tau$. As an example of application, the program used to generate this figure can be found as a supplementary material. Both curves are fitted at half height and at that point they perfectly match, however, they slightly differ at the base of the curve. Figure 4 shows a comparison, using the EGH model, of three different values of $\tau$ for a given value of $T_{p}, \sigma$, height and heating rate, and it reflects how the value of $\tau$ is related to the skewness of the curve, the more negative is $\tau$, the more skewed is the curve; the chosen values of $\tau$ are negative representing a curve skewness to the left. Figure 5 shows in which way the EGH parameters translate into the $n$ thorder Arrhenius ones ( $n, E$, and $A$ ), for given values of heating rate, $T_{p}$ and $\sigma$, and changing values of $\tau$; the three kinetic parameters decrease with a more negative $\tau$, meaning that the values of the kinetic parameters decrease as the curve is more skewed to the left.

\subsubsection{Estimation of the biomass relative composition for nth-order}

The fraction of each component that can be determined from a DTG curve is their corresponding contribution to the volatiles released. The height of the peaks is related to the component fractions, for a $d \alpha / d T$ peak, according to

$$
x=H \frac{\beta}{A} e^{T_{E} / T_{p}}\left[1+(n-1) \frac{A}{\beta} \bar{p}\left(T_{p}\right)\right]^{-n /(1-n)}
$$

In this section, mathematical relationships have been given between parameters that characterize the shape of a peak in a DTG curve and the parameters of an $n$-order reaction kinetics model. In Sec. 3.2, we adapt the found relationships for a first order reaction model. 
For a first-order reaction model, $n=1$, and $f(\alpha)=(1-\alpha)$. The expressions for $\sigma, \tau$, and the pre-exponential factor found for $n$ th-order given in Sec. 3.1 simplify to

$$
\begin{aligned}
\sigma & \approx \frac{T_{p}^{2}}{T_{E}} \\
\tau & \approx-\frac{2}{3} \frac{T_{p}^{2}}{T_{E}} \\
A & =\beta \frac{T_{E}}{T_{p}^{2}} e^{T_{E} / T_{p}} .
\end{aligned}
$$

It is worth noting that this
its corresponding form.

Assuming an Arrhenius rate constant, the integral $\bar{p}(T)$ in Eq. (4) would be defined as $\bar{p}(T)=T_{E} p\left(T_{E} / T\right)$, where

$$
p(y)=-\int_{y}^{T_{E} / T_{0}} d y \frac{e^{-y}}{y^{2}}
$$

For first order, the integral in Eq. (22) is now modified to $g(\alpha)=-\ln (1-\alpha)$, therefore the expression for conversion is

$$
(1-\alpha)=\exp \left[-\left(-\frac{T_{E}}{T_{p}}\right)^{2} e^{T_{E} / T_{p}} p\left(T_{E} / T\right)\right]
$$

Incorporating this expression, as well as the Arrhenius rate constant and the expressions found for $\sigma$ and $A$ into Eq. (3), we obtain the following integrated form of the reation rate, which is for a first order reaction model using an Arrhenius reaction rate

$$
\frac{d \alpha}{d T}=-\exp \left[\frac{T_{p}}{\sigma}-\frac{T_{p}^{2}}{\sigma T}-\left(\frac{T_{p}}{\sigma}\right)^{2} e^{T_{p} / \sigma} p\left(T_{E} / T\right)\right] \sigma^{-1}
$$

The parameters for the peak shape can be converted to the parameters for the kinetics model with Eq. (41) and Eq. (42):

$$
\begin{aligned}
& E=R \frac{T_{p}^{2}}{\sigma} \\
& A=\frac{\beta}{\sigma} e^{T_{p} / \sigma}
\end{aligned}
$$

According to these expressions, $E$ and $A$ are inversely proportional to $\sigma$, and their values increase with increasing $T_{p}$. 
The height of the peak is particularly useful when dealing with multi-component mechanisms, as explained in Sec. 3.2.1. The height of the peak is found when $T=T_{p}$ :

$$
H_{p, i}=\exp \left[-\left(\frac{T_{p, i}}{\sigma}\right)^{2} e^{T_{p, i} / \sigma_{i}} p\left(T_{E} / T_{p, i}\right)\right] x_{i} \beta \sigma_{i}^{-1}
$$

\subsubsection{Estimation of the biomass relative composition for first order}

The relative contribution of cellulose, hemicellulose, and lignin to the volatile yield can be estimated from the peak heights $H_{p}$ of a DTG curve. For a multi-component kinetic mechanism, the total decomposition of the biomass is a sum of the decomposition of its components

$$
\frac{d \alpha}{d t}=\sum_{i=1}^{n} x_{i} \frac{d \alpha_{i}}{d t}
$$

where $x_{i}$ is the fraction of component $i$. From the height equation, Eq. (43), the component fraction is given by

$$
x_{i}=\frac{H_{p, i} \sigma_{i}}{\beta \exp \left[-\left(\frac{T_{p, i}}{\sigma_{i}}\right)^{2} e^{T_{p, i} / \sigma_{i}} p\left(T_{E, i} / T\right)\right]}
$$

If fitting the experimental data, the composition calculated can be used as an initial estimation for the components fractions, to be optimized along with the values of $T_{p}$ and $\sigma$.

\subsection{Kinetic analysis of experimental data from different heating rates}

Thermogravimetric data from the same biomass sample, will have different peak positions depending on the heating rate. When considering the decomposition of a single component, the kinetic parameters, in principle, could be calculated by adapting the methodology presented previously to deal with different heating rates, by equating the expression for the pre-exponential factor, found in Eq. (32) for an $n$ th-order reaction, from two different heating rates

$$
-\ln \frac{\beta}{\beta^{*}}=\frac{T_{E}}{T_{p}^{*}}\left(\frac{T_{p}^{*}}{T_{p}}-1\right)+2 \ln \left(\frac{T_{p}^{*}}{T_{p}}\right)
$$

and a simplified form with only peak shape parameters for first-order

$$
-\ln \frac{\beta}{\beta^{*}}=\frac{T_{p}^{*}}{\sigma^{*}}\left(\frac{T_{p}^{*}}{T_{p}}-1\right)+2 \ln \left(\frac{T_{p}^{*}}{T_{p}}\right)
$$

where the asterisk corresponds to parameters of the reference conditions. Ideally, given a single peak, for each pair of heating rates, the same activation energy should be found, an example of the application of this equation is given in 
Sec. 4.2.1

\section{Results and Discussion}

To illustrate the utility of the expressions developed in the previous sections, specific examples of the kinetic analysis of thermogravimetric data from biomass pyrolysis are provided, where the reactions are assumed to proceed with an Arrhenius rate constant and to be either a first-order or $n$ th-order model. In Sec. 4.1, experimental data from a single heating rate experiment are analyzed, and, in Sec. 4.2, data from several heating rates are evaluated simultaneously. For both cases, the biomass is initially treated as a single component (e.g., pure cellulose). Then it is considered to be a combination of its main chemical components (i.e. hemicellulose, cellulose and lignin).

\subsection{Kinetic analysis of experimental data from a single heating rate}

\subsubsection{Single-component mechanism}

Experimental TGA data of cellulose pyrolysis at $10 \mathrm{~K} \mathrm{~min}^{-1}$ [34] has been used as an example of kinetic analysis by directly calculating the kinetic parameters from only the measurable features of the peak shape, without the need of performing a fit. The shape parameters are measured on the DTG curve of conversion versus temperature, which area is one, therefore $T_{p}, \sigma, \tau$, and height and conversion at the $T_{p}$ are quantitatively estimated. The kinetic parameters for a first order reaction with an Arrhenius rate constant are $E$ and $A$, and for an $n$ th-order reaction model are $E, A$, and $n$. The number of peak shape parameters to be measured depends on the number of kinetic parameters to be calculated for the chosen reaction model. For the peak in Figure 6, the observed shape parameters are $T_{p}=632.4 \mathrm{~K}$, $W_{-}=22.9 \mathrm{~K}, W_{+}=15.6 \mathrm{~K}, H=0.024 \mathrm{~K}^{-1}$ and $\alpha=0.616$; the corresponding values of $\sigma$ and $\tau$ are $16.0 \mathrm{~K}$ and $-10.4 \mathrm{~K}$, respectively.

Assuming a first-order reaction model, Eqs. (41) and (42) are used to directly calculate the values of the kinetic parameters, resulting in $E=206.3 \mathrm{~kJ} \mathrm{~mol}^{-1}$ and $A=1.2 \times 10^{15} \mathrm{~s}^{-1}$, similar to the literature values of $E=237 \mathrm{~kJ} \mathrm{~mol}^{-1}$ and $A=1 \times 10^{18} \mathrm{~s}^{-1}$ [34]. In this case, the shape parameters that are constrained are $T_{p}$ and $\sigma$. Assuming an $n$ thorder reaction model, Eqs. (28), (30), (31), (32), and (33) can be used to calculate the kinetic parameters. Each expression constrains one of the measurable parameters, but in this case only three of the expressions are needed in order to calculate $E, A$, and $n$. In this example, we choose to constrain $T_{p}, \sigma$, and $\tau$, and, consequently, we obtain $E=194.9 \mathrm{~kJ} \mathrm{~mol}^{-1}, A=1.3 \times 10^{14} \mathrm{~s}^{-1}$, and $n=0.94$. Calculating the height $\left(H_{p}=0.024 \mathrm{~K}^{-1}\right)$ and conversion ( $\alpha=0.625$ ) from the resulting kinetic values, it can be noticed that the resulting height coincides with the measured one, but the conversion is slightly bigger than observed, which makes sense if we compare it with the given range of conversion values for a first-order reaction model [39] and we see that our resulting order of reaction is slightly smaller 
than one, and conversion at the peak is slightly bigger than the literature value. The height and conversion could have also been used to calculate the kinetic parameters, instead of $\sigma$ and $\tau$; the only difference is which observable characteristics of the peak shape we want to predict with more accuracy.

\subsubsection{Multi-component mechanism}

Experimental thermogravimetric data of beech wood pyrolysis at $5 \mathrm{~K} \mathrm{~min}^{-1}$ [15] were used as an example of for the deconvolution of a multi-component system in order to determine not only the reaction kinetics, but also the composition of the sample. The fitting procedure of a DTG curve consists of performing a least-squares minimization of the difference between the experimental and predicted rate of mass loss:

$$
\begin{aligned}
\text { O.F. } & =\sum_{k=1}^{n}\left[\left(\frac{d \alpha}{d T}\right)_{\text {calc }}-\left(\frac{d \alpha}{d T}\right)_{\text {exp }}\right]^{2} \\
\left(\frac{d \alpha}{d T}\right)_{\text {calc }} & =\sum_{i=1}^{3} x_{i} \frac{d \alpha_{i}}{d T}
\end{aligned}
$$

For a first-order reaction model, the rate of reaction is calculated with Eq. (40) with respect to time. The input values are the initial estimates for the parameters to adjust, determined by direct observation of the DTG curve, these parameters are the $T_{p, i}, \sigma_{i}$, and $H_{p, i}$, where $i$ denotes each component reaction. The biomass relative composition $x_{i}$ is estimated with Eq. (45), and the parameters to be optimized are $T_{p, i}, \sigma_{i}$, and $x_{i}$. The resulting values of $T_{p, i}$ and $\sigma_{i}$ can be used in Eqs. (41) and (42) to obtain the values of activation energy and pre-exponential factor.

The peak temperatures were allowed to vary in a range of $50 \mathrm{~K}$ from the selected temperature, this range depends on how distinguishable the curve peaks are and their level of overlapping, in this case, for cellulose and hemicellulose, their $T_{p}$ was fairly identifiable but not so much for lignin. The $\sigma$ was allowed to vary between $0.5 \sigma$ and $1.5 \sigma$. Individual bounds for a particular component can be specified, but yet, provided a good initial guess, the ones that are clearly around the value given, will tend to remain there independently of the size of the range. The resulting fit is shown in Figure 7, and the resulting kinetic parameters and composition in Table 1. For deconvolution with the $n$ th-order reaction model, the fit is done directly with the EGH model, setting the bounds for $\tau$ and $\sigma$ as $(0.5 \tau$ to $1.5 \tau)$ and $(0.5 \sigma$ to $1.5 \sigma)$, respectively. In this case, the initial conditions are the resulting parameters found from the deconvolution with a first-order reaction. The resulting kinetic parameters for the first-order reaction model are similar to those reported in the literature, and the calculated kinetic parameters for the $n$ th-order reaction model, which can also be found in Table 1, are not far from the values determined for the first-order reaction.

The determined relative contribution of the biomass components is between $34.6 \%$ and $41.2 \%$ hemicellulose, between 45.8 and $55.7 \%$ cellulose and between 9.7 and $13 \%$ lignin. The reported composition, experimentally de- 
Table 1: Resulting kinetic parameters from deconvolution of beech wood as in Figure 7.

\begin{tabular}{lccc}
\hline \hline & first-order & $n$ th-order & literature [15] \\
\hline$E_{H C E} / \mathrm{kJ} \mathrm{mol}^{-1}$ & 97 & 114.8 & 100 \\
$A_{H C E} / \mathrm{s}^{-1}$ & $2.4 \times 10^{6}$ & $1.5 \times 10^{8}$ & $4.3 \times 10^{6}$ \\
$E_{C E L L} / \mathrm{kJ} \mathrm{mol}^{-1}$ & 231.5 & 191.5 & 236 \\
$A_{C E L L} / \mathrm{s}^{-1}$ & $1.7 \times 10^{17}$ & $6.3 \times 10^{13}$ & $3.8 \times 10^{17}$ \\
$E_{L I G} / \mathrm{kJ} \mathrm{mol}^{-1}$ & 45.9 & 74.3 & 46 \\
$A_{L I G} / \mathrm{s}^{-1}$ & 5.5 & $1.5 \times 10^{3}$ & 4 \\
$n_{H C E}$ & 1 & 1.3 & 1 \\
$n_{C E L L}$ & 1 & 0.83 & 1 \\
$n_{L I G}$ & 1 & 1.2 & 1 \\
$\chi^{2}$ & 0.011 & 0.005 & \\
\hline \hline
\end{tabular}

Table 2: Resulting kinetic parameters from simultaneous deconvolution of macadamia nutshell as in Figure 9.

\begin{tabular}{lccc}
\hline \hline & first-order & $n$ th-order & literature [45] \\
\hline$E_{H C E} / \mathrm{kJ} \mathrm{mol}^{-1}$ & 125.9 & 131.4 & $132.2-150.6$ \\
$A_{H C E} / \mathrm{s}^{-1}$ & $1.9 \times 10^{9}$ & $6.6 \times 10^{9}$ & $2.9 \times 10^{9}-1.8 \times 10^{11}$ \\
$E_{C E L L} / \mathrm{kJ} \mathrm{mol}^{-1}$ & 182.1 & 173.1 & $221.2-265.7$ \\
$A_{C E L L} / \mathrm{s}^{-1}$ & $6.2 \times 10^{12}$ & $1.1 \times 10^{12}$ & $8.9 \times 10^{15}-8.2 \times 10^{19}$ \\
$E_{L I G} / \mathrm{kJ} \mathrm{mol}^{-1}$ & 59.2 & 72.7 & $62.8-74.5$ \\
$A_{L I G} / \mathrm{s}^{-1}$ & $1.3 \times 10^{2}$ & $1.9 \times 10^{3}$ & $2.7 \times 10^{2}-1 \times 10^{4}$ \\
$n_{H C E}$ & 1 & 1.1 & 1 \\
$n_{C E L L}$ & 1 & 0.88 & 1 \\
$n_{L I G}$ & 1 & 1.2 & 1 \\
$\chi^{2}$ & 0.036 & 0.028 & \\
\hline \hline
\end{tabular}

termined for beech [43], is $78 \%$ of holocellulose (hemicellulose + cellulose), $20 \%$ of lignin, and $2 \%$ of extractives on a dry basis. Taking into account that lignin is the major contributor to char formation [44], the calculated results seem consistent with the experimental data, since in the volatile there is a bigger fraction of holocellulose than in the raw biomass. In the case of deconvolution of DTG data from a single heating rate, it has been found that there is no significant difference or improvement when considering an $n$ th-order reaction model instead of a first-order one.

Fitting is a mathematical procedure that does not obey any thermo-chemical law, and sometimes results that are not in agreement with what we already know about the thermal behavior of the material can be obtained. For instance, in the case example, we need to make sure that the peak temperatures and decomposition ranges are in agreement with what is reported in the literature for similar conditions. The advantage of using the peak shape methodology, is the easy estimation of the initial values to be adjusted, and that their constraints have the same units as in the DTG curve axis. For instance, if we observe that the predicted decomposition does not take place in a temperature range in agreement with the ranges reported in the literature, the fit could be adjusted by shifting the $T_{p}$ towards the reported 
range or modifying the bounds to widen them, symmetrically or not.

\subsection{Kinetic analysis of experimental data from several heating rates}

\subsubsection{Single-component mechanism}

Experimental data from cellulose pyrolysis at different heating rates $[34,46,47,48,49,19,21,20,50]$ were used to exemplify how the peak shape method can be used to find a single set of kinetic parameters across all the DTG experimental data. For DTG data from a single component at different heating rates, Eq. (47) could be used to find a single set of kinetic parameters, as rendered in Figure 8a, where each line in the plot corresponds to a different activation energy. The peak shape parameter obtained from the experimental data is the $T_{p}$ from DTG curves of cellulose pyrolysis at different heating rates, and is represented by black dots. For the sake of clarification, the red crosses correspond to calculated data derived for $E=100 \mathrm{~kJ} \mathrm{~mol}^{-1}$ at three different heating rates, their DTG curves are shown in Figure 8b.

If the experimental data would display an ideal behavior, as the calculated data, all points would fall on the same line, defining which is the specific activation energy for cellulose, but as can be observed from the figure, the experimental data does not behave as expected.

There could be several explanations for the fact that the experimental data does not define a unique activation energy. One explanation is related to the comparability of the experimental data, it is possible that the different cellulose sources used by the different authors, and the different experimental conditions might have had an impact on the distribution of the peak temperatures, or that the experiments might be governed by transport phenomena rather than being kinetically controlled; furthermore, it is also possible that the single-step and first-order reaction model does not satisfactorily render the complexity of the decomposition process. The experimental points allow the delineation of a range of activation energies, but to find a single set of kinetic parameters suitable across the range of studied temperatures, a simultaneous evaluation of thermogravimetric data at different heating rates is considered necessary and will be discussed in Sec. 4.2.2.

In Figure 8a, at the bottom, the width of the peak is plotted with respect to the heating rate. From both plots in Figure 8a, it can be observed that $T_{p}$ and $\sigma$ increase proportionally with the heating rate, following the expression

$$
\frac{\sigma}{\sigma^{*}}=\left(\frac{T_{p}}{T_{p}^{*}}\right)^{2}
$$

In the following section, we use the peak shape method to perform a simultaneous evaluation of DTG data from several heating rates with a multi-component mechanism. 


\subsubsection{Multi-component mechanism}

Experimental data from pyrolysis of macadamia nut shell are taken from the literature [45] to serve as an example of simultaneous deconvolution of several DTG curves at different heating rates, each of them featuring three main peaks corresponding to hemicellulose, cellulose and lignin. The choice of the reference curve is arbitrary. From four DTG curves of biomass pyrolysis performed at heating rates of $5,10,20$, and $30 \mathrm{~K} \mathrm{~min}^{-1}$, the one at $10 \mathrm{~K} \mathrm{~min}^{-1}$ was selected to be a reference curve. This simultaneous fitting is suitable for single and multi-component decomposition mechanisms, but in the following example, we are going to consider a multi-component mechanism, the more complex of the two.

The input parameters for a first-order reaction model are $T_{p, i}^{*}, \sigma_{i}^{*}$ and $H_{p, i}^{*}$, where the asterisk indicates that the properties belong to the reference curve. The initial values for the peak temperature and width are estimated by direct observation of the DTG curve, and the initial estimation of the composition comes from applying Eq. (45) to the reference curve. From the fitting, a single composition and a single set of kinetic parameters able to render the biomass decomposition, taking into account the impact of different heating rates, is obtained.

The fitting procedure is similar to the one applied in Sec. 4.1.2 for a single heating rate. The difference in this case is that a reference curve is chosen, and the rest of curves at the different heating rates are calculated with Eq. (47) with respect to the reference. In this way, only the kinetic parameters for the reference curve, $T_{p, i}^{*}, \sigma_{i}^{*}$ and $x_{i}^{*}$, need to be adjusted to minimize the objective function

$$
\begin{aligned}
\text { O.F. } & =\sum_{k=1}^{n}\left[\left(\frac{d \alpha}{d T}\right)_{\text {calc }}-\left(\frac{d \alpha}{d T}\right)_{\exp }\right]^{2} \\
\left(\frac{d \alpha}{d T}\right)_{\text {calc }} & =\sum_{j=1}^{4} \sum_{i=1}^{3} x_{i}^{(j)} \frac{d \alpha_{i}^{(j)}}{d T}
\end{aligned}
$$

where $j$ denotes the different heating rates, and $i$ denotes the components in the biomass.

The bounds for $T_{p}$ are $50 \mathrm{~K}$ on each side of the chosen temperature value, tha range is large due the fact that for hemicellulose and lignin the peaks are overlapped and difficult to identify, the lower bound for $\sigma$ is 0.5 of the initial value, and the upper bound is 1.5 times the initial value. The resulting fit can be found in Figure 9 and the resulting kinetic parameters in Table 2.

Deconvolution of the DTG curve with an $n$ th-order reaction mechanism was also carried out, and the results are reported in Table 2. Similar to the deconvolution performed in Sec. 4.1.2, no significant improvement in the fit was found by using an $n$ th-order reaction model rather than the simpler first-order model. The estimated composition of the biomass is $27.1 \%$ hemicellulose, between 51.7 and $53.4 \%$ cellulose, and between 19.5 and $21.1 \%$ lignin. In comparison with the expreimentally estimated raw material composition [51], the fraction of cellulose and hemicel- 
lulose is larger with a lower fraction of lignin, like in the previous example given in Sec. 4.1.2. The calculated kinetic parameters are similar to but slightly differ from the reported literature ranges, which might be due to the assumption of only three components and leaving out extractives.

The reliability of the calculated kinetic parameters depends on the initial values and bound of the fit parameters, the suitability of the chosen kinetic mechanism and the quality of the experimental data, meaning that the experimental conditions should ensure that the process takes place in a kinetically controlled regime.

\section{Conclusions}

In this paper, we develop a method to estimate the parameters of a reaction kinetics model, directly from the shape of the derivative thermogravimetric (DTG) curve. It is suitable for single or multi-component mechanisms involving single-step reactions. The present work comprehensively encompasses the measurable shape parameters of the DTG curve of biomass pyrolysis and from the study of chromatography peaks reported in the literature, by deriving mathematical expressions that directly link the peak shape with kinetics. The shape parameters reported in this work can be selected and used in any combination, according to the number of required kinetic parameters specific for each reaction model. This gives the flexibility to decide which are the decomposition characteristics that we want to predict with more accuracy, and also to assume different rate constants and reaction models.

In the specific examples of application included in this work, an Arrhenius rate constant and a first $/ n$ th-order reaction model are used, which requires two/three kinetic parameters to be determined. The results of the deconvolution examples, show how the shape method allows for an easy estimation of the initial values of the parameters, which can be obtained by direct observation of the DTG curve. Furthermore, the bounds of the parameters can also be defined from direct observation, in order to improve the fit or reduce the convergence time. When dealing with singlecomponent mechanisms, in which the DTG curve displays a single peak, the kinetic parameters can be calculated by directly applying the expressions that link the peak shape parameters with the reaction kinetics, without the need to fit. For multi-component mechanisms in which the DTG displays multiple peaks, the goodness of the fit relies on the precision of the initial values and bounds of the fit parameters, and their estimation depends on the overlapping degree of the peaks. The resulting kinetic parameters and contribution fractions of the biomass main components, could then be used as the initial values of the key kinetic parameters and provide reasonable constraints in order to reduce the computational cost of more elaborate fitting procedures. To improve the fit for the given examples, other components could be included in the kinetic mechanism, such as extractives, and differing reaction models from the $n$ th-order could be considered. The present method for the kinetic analysis of thermogravimetric data could also be applied to other similar thermal reactions from which we can obtain a DTG curve, such as a drying process or the 
decomposition of other polymers or solid fuels.

346 Supplementary material

Program from Figure 3 written in Python 2.7.

\section{Acknowledgements}

349 The authors gratefully aknowledge the finantial support from the Scottish Funding Council - Global Challenge 350 Research Fund and the Biofuels Research Infrastructure for Sharing Knowledge (BRISK). 
${ }_{352} \alpha \quad$ Conversion fraction

${ }_{353} \beta \quad$ Heating rate $\left(\mathrm{K} \mathrm{s}^{-1}\right)$

${ }_{354} \sigma \quad$ Width of the peak as in a Gaussian model (K)

355 $\theta \quad$ Fraction of the peak height

${ }_{356} \tau \quad$ Related to the asymmetry of the peak $(\mathrm{K})$

${ }_{357} \chi^{2} \quad$ chi-square

${ }_{358} \mathrm{x}_{i} \quad$ Contribution fraction of the component $i$ to the volatile yield

359 A Pre-exponential factor $\left(\mathrm{s}^{-1}\right)$

${ }_{360} \mathrm{E} \quad$ Activation energy $\left(\mathrm{kJ} \mathrm{mol}^{-1}\right)$

${ }_{361} \mathrm{~g}(\alpha) \quad$ Integral of the differential conversion versus the reaction model

${ }_{362} \mathrm{H}_{p} \quad$ Height of the peak at the peak temperature $\left(\mathrm{s}^{-1}\right.$ or $\left.\mathrm{K}^{-1}\right)$

$363 \mathrm{k} \quad$ Rate constant $\left(\mathrm{s}^{-1}\right)$

${ }_{364} \mathrm{~m}_{0} \quad$ Initial mass (units of mass)

$365 \quad \mathrm{~m}_{f} \quad$ Final mass of the sample (units of mass)

${ }_{366} \mathrm{~m}_{t} \quad$ Mass of the sample at a given time (units of mass)

${ }_{367} \mathrm{p}(\mathrm{y}) \quad$ Exponential integral of the Arrhenius equation

${ }_{368} \mathrm{R} \quad$ Universal gas constant $\left(8.314 \cdot 10^{-3} \mathrm{~kJ} \mathrm{~K}^{-1} \mathrm{~mol}^{-1}\right)$

${ }_{369} \mathrm{~T}_{E} \quad$ Auxiliary parameter, $\mathrm{E} / \mathrm{R}(\mathrm{K})$

${ }_{370} \mathrm{~T}_{p} \quad$ Peak temperature, temperature at the maximum rate of reaction $(\mathrm{K})$

${ }_{371} \quad \mathrm{~W}_{-} \quad$ Width from the center to the left of the peak $(\mathrm{K})$

$372 \quad \mathrm{~W}_{+} \quad$ Width from the center to the right of the peak $(\mathrm{K})$

373 RMS Root mean square 
[1] A. Molino, V. Larocca, S. Chianese, D. Musmarra, Biofuels production by biomass gasification: A review, Energies 11 (4) (2018) 811. doi:10.3390/en11040811.

URL http://dx.doi.org/10.3390/en11040811

[2] S. Wang, X. Guo, K. Wang, Z. Luo, Influence of the interaction of components on the pyrolysis behavior of biomass, J. Anal. Appl. Pyrolysis 91 (1) (2011) 183-189. doi:10.1016/j.jaap.2011.02.006.

[3] P. Giudicianni, G. Cardone, G. Sorrentino, R. Ragucci, Hemicellulose, cellulose and lignin interactions on arundo donax steam assisted pyrolysis, J. Anal. Appl. Pyrolysis 110 (Supplement C) (2014) 138 - 146. doi:10.1016/j.jaap.2014.08.014.

[4] J. Yu, N. Paterson, J. Blamey, M. Millan, Cellulose, xylan and lignin interactions during pyrolysis of lignocellulosic biomass, Fuel 191 (Supplement C) (2017) 140 - 149. doi:10.1016/j.fuel.2016.11.057.

[5] C. Koufopanos, A. Lucchesi, G. Maschio, Kinetic modelling of the pyrolysis of biomass and biomass components, Can. J. Chem. Eng. 67 (1) (1989) 9. doi:10.1002/cjce.5450670111.

[6] P. Harmsen, W. Huijgen, L. Bermudez, R. Bakker, Literature review of physical and chemical pretreatment processes for lignocellulosic biomass, Tech. rep., Wageningen UR Food \& Biobased Research (2010).

[7] J. S. Tumuluru, C. T. Wright, R. D. Boardman, R. J. Hess, S. Sokhansanj, Review on biomass torrefaction process and product properties and design of moving bed torrefaction system model development, in: 2011 Louisville, Kentucky, August 7-10, 2011, American Society of Agricultural and Biological Engineers, 2011, p. 1.

[8] T. Martí-Rosselló, J. Li, L. Lue, Kinetic models for biomass pyrolysis., Archives of Industrial Biotechnology 1 (1).

[9] P. Basu, Biomass Gasification, Pyrolysis and Torrefaction: Practical Design and Theory, Elsevier Science, 2013.

[10] P. Basu, Chapter 3 - Pyrolysis and Torrefaction, Academic Press, Boston, 2010, book section 3, pp. 65-96.

[11] A. Demirbas, G. Arin, An overview of biomass pyrolysis, Energy Sources 24 (5) (2002) 471-482. doi:10.1080/00908310252889979.

[12] A. Bridgwater, Principles and practice of biomass fast pyrolysis processes for liquids, J. Anal. Appl. Pyrolysis 51 (1) (1999) 3 - 22. doi:10.1016/S0165-2370(99)00005-4.

[13] E. Ranzi, A. Cuoci, T. Faravelli, A. Frassoldati, G. Migliavacca, S. Pierucci, S. Sommariva, Chemical kinetics of biomass pyrolysis, Energy Fuels 22 (6) (2008) 4292-4300. doi:10.1021/ef800551t.

[14] E. Ranzi, P. E. A. Debiagi, A. Frassoldati, Mathematical modeling of fast biomass pyrolysis and bio-oil formation. note i: Kinetic mechanism of biomass pyrolysis, ACS Sustainable Chemistry \& Engineering 5 (4) (2017) 2867-2881. doi:10.1021/acssuschemeng.6b03096.

[15] M. G. Grønli, G. Várhegyi, C. Di Blasi, Thermogravimetric analysis and devolatilization kinetics of wood, Ind. Eng. Chem. Res. 41 (17) (2002) 4201-4208. doi:10.1021/ie0201157.

[16] C. Branca, A. Albano, C. Di Blasi, Critical evaluation of global mechanisms of wood devolatilization, Thermochim Acta 429 (2) (2005) 133-141. doi:10.1016/j.tca.2005.02.030.

[17] X. Li, C. Sun, B. Zhou, Y. He, Determination of hemicellulose, cellulose and lignin in moso bamboo by near infrared spectroscopy, Sci. Rep. 5 (2015) 17210. doi:10.1038/srep17210.

[18] L. Burhenne, J. Messmer, T. Aicher, M.-P. Laborie, The effect of the biomass components lignin, cellulose and hemicellulose on $\{$ TGA $\}$ and fixed bed pyrolysis, J. Anal. Appl. Pyrolysis 101 (2013) 177 - 184. doi:10.1016/j.jaap.2013.01.012.

[19] M. Carrier, A. Loppinet-Serani, D. Denux, J.-M. Lasnier, F. Ham-Pichavant, F. Cansell, C. Aymonier, Thermogravimetric analysis as a new method to determine the lignocellulosic composition of biomass, Biomass Bioenergy 35 (1) (2010) $298-307$. doi:10.1016/j.biombioe.2010.08.067.

[20] V. Cozzani, A. Lucchesi, G. Stoppato, G. Maschio, A new method to determine the composition of biomass by thermogravimetric analysis., Can. J. Chem. Eng. 75 (1) (1997) 6. doi:10.1002/cjce.5450750120. 
[21] K. Singh, M. Risse, K. C. Das, J. Worley, Determination of composition of cellulose and lignin mixtures using thermogravimetric analysis, J. Energy Res. Technol. 131 (2). doi:10.1115/1.3120349.

[22] T. Reed, A. Das, Handbook of biomass downdraft gasifier engine system.

[23] A. I. Ferreiro, M. Rabaal, M. Costa, A combined genetic algorithm and least squares fitting procedure for the estimation of the kinetic parameters of the pyrolysis of agricultural residues, Energy Conversion and Management 125 (2016) 290 - 300, sustainable development of energy, water and environment systems for future energy technologies and concepts. doi:https://doi.org/10.1016/j.enconman.2016.04.104. URL http://www.sciencedirect.com/science/article/pii/S0196890416303648

[24] L. Xu, Y. Jiang, L. Wang, Thermal decomposition of rape straw: Pyrolysis modeling and kinetic study via particle swarm optimization, Energy Conversion and Management 146 (2017) 124 - 133. doi:https://doi.org/10.1016/j.enconman.2017.05.020. URL http://www.sciencedirect.com/science/article/pii/S019689041730451X

[25] J. H. Flynn, L. A. Wall, General treatment of the thermogravimetry of polymers, J. Res. Nat. Bur. Stand. 70 (6) (1966) $487-523$. doi:10.6028/jres.070a.043.

[26] D. W. Van Krevelen, K. Te Nijenhuis, Properties of polymers: their correlation with chemical structure; their numerical estimation and prediction from additive group contributions, Elsevier, 2009.

[27] H. E. Kissinger, Variation of peak temperature with heating rate in differential thermal analysis, J. Res. Nat. Bur. Stand. 57 (4) (1956) $217-221$. doi:10.6028/jres.057.026.

[28] S. Kim, J. K. Park, Characterization of thermal reaction by peak temperature and height of dtg curves, Thermochimica Acta 264 (1995) 137 - 156. doi:https://doi.org/10.1016/0040-6031(95)02316-T. URL http://www.sciencedirect.com/science/article/pii/004060319502316T

[29] S. Kim, E.-S. Jang, D.-H. Shin, K.-H. Lee, Using peak properties of a dtg curve to estimate the kinetic parameters of the pyrolysis reaction: application to high density polyethylene, Polym. Degrad. Stab. 85 (2) (2004) 799-805. doi:10.1016/j.polymdegradstab.2004.03.009.

[30] D. Dollimore, T. Evans, Y. Lee, F. Wilburn, Correlation between the shape of a TG/DTG curve and the form of the kinetic mechanism which is applying, Thermochimica Acta (2) 249-257. doi:10.1016/0040-6031(92)85081-6.

[31] S. Vyazovkin, Kinetic concepts of thermally stimulated reactions in solids: a view from a historical perspective, Int. Rev. Phys. Chem. 19 (1) (2000) 45-60. doi:10.1080/014423500229855.

[32] S. Vyazovkin, A. K. Burnham, J. M. Criado, L. A. Pérez-Maqueda, C. Popescu, N. Sbirrazzuoli, Ictac kinetics committee recommendations for performing kinetic computations on thermal analysis data, Thermochim. Acta 520 (1) (2011) 1-19. doi:10.1016/j.tca.2011.03.034.

[33] G. Várhegyi, Aims and methods in non-isothermal reaction kinetics, J. Anal. Appl. Pyrolysis 79 (1) (2007) 278-288. doi:10.1016/j.jaap.2007.01.007.

[34] M. J. Antal, G. Varhegyi, E. Jakab, Cellulose pyrolysis kinetics: revisited, Ind. Eng. Chem. Res. 37 (4) (1998) $1267-1275$. doi:10.1021/ie970144v.

[35] A. Khawam, D. R. Flanagan, Solid-state kinetic models: basics and mathematical fundamentals, J. Phys. Chem. B 110 (35) (2006) 1731517328, pMID: 16942065. doi:10.1021/jp062746a.

[36] J. Blondeau, H. Jeanmart, Biomass pyrolysis at high temperatures: Prediction of gaseous species yields from an anisotropic particle, Biomass Bioenergy 41 (2012) 107-121. doi:10.1016/j.biombioe.2012.02.016.

[37] K. Lan, J. W. Jorgenson, A hybrid of exponential and gaussian functions as a simple model of asymmetric chromatographic peaks, J. Chromatogr. A 915 (1) (2001) 1 - 13.

[38] J. López-Beceiro, A. Álvarez-García, T. Sebio-Puñal, S. Zaragoza-Fernández, B. Álvarez-García, A. Díaz-Díaz, J. Janeiro, R. Artiaga, Kinetics of thermal degradation of cellulose: Analysis based on isothermal and linear heating data, BioResources 11 (3). 
[39] C. Haixiang, L. Naian, Z. Weitao, Critical study on the identification of reaction mechanism by the shape of TG/DTG curves, Solid State Sciences (4) 455-460. doi:10.1016/j.solidstatesciences.2009.12.007.

[40] C. Di Blasi, Modeling chemical and physical processes of wood and biomass pyrolysis, Prog. Energy Combust. Sci. 34 (1) (2008) 47-90.

[41] H. E. Kissinger, Reaction kinetics in differential thermal analysis, Anal. Chem. 29 (11) (1957) 1702-1706. doi:10.1021/ac60131a045.

[42] R. L. Blaine, H. E. Kissinger, Homer kissinger and the kissinger equation, Thermochim. Acta 540 (2012) 1-6. doi:10.1016/j.tca.2012.04.008.

[43] C. Di Blasi, C. Branca, A. Santoro, R. A. P. Bermudez, Weight loss dynamics of wood chips under fast radiative heating, J. Anal. Appl. Pyrolysis 57 (1) (2001) 77-90. doi:10.1016/s0165-2370(00)00119-4.

[44] B. Cagnon, X. Py, A. Guillot, F. Stoeckli, G. Chambat, Contributions of hemicellulose, cellulose and lignin to the mass and the porous properties of chars and steam activated carbons from various lignocellulosic precursors, Bioresour. Technol. 100 (1) (2009) 292 - 298.

[45] T. Xavier, T. Lira, M. Schettino Jr, M. Barrozo, A study of pyrolysis of macadamia nut shell: Parametric sensitivity analysis of the ipr model, Braz. J. Chem. Eng. 33 (1) (2016) 115-122. doi:10.1590/0104-6632.20160331s00003629.

[46] H. Zhou, Y. Long, A. Meng, S. Chen, Q. Li, Y. Zhang, A novel method for kinetics analysis of pyrolysis of hemicellulose, cellulose, and lignin in tga and macro-tga, RSC Advances 5 (34) (2015) 26509-26516. doi:10.1039/c5ra02715b.

[47] Y.-C. Lin, J. Cho, G. A. Tompsett, P. R. Westmoreland, G. W. Huber, Kinetics and mechanism of cellulose pyrolysis, J. Phys. Chem. C 113 (46) (2009) 20097-20107. doi:10.1021/jp906702p.

[48] S. Wang, Q. Liu, K. Wang, X. Guo, Z. Luo, K. Cen, T. Fransson, Study on catalytic pyrolysis of manchurian ash for production of bio-oil, Int. J. Green Energy 7 (3) (2010) 300-309. doi:10.1080/15435071003796111.

[49] K. M. Isa, S. Daud, N. Hamidin, K. Ismail, S. A. Saad, F. H. Kasim, Thermogravimetric analysis and the optimisation of biooil yield from fixed-bed pyrolysis of rice husk using response surface methodology (rsm), Ind. Crops Prod. 33 (2) (2011) $481-487$. doi:10.1016/j.indcrop.2010.10.024.

[50] S. Wang, Q. Liu, Z. Luo, L. Wen, K. Cen, Mechanism study on cellulose pyrolysis using thermogravimetric analysis coupled with infrared spectroscopy, Front. Energy Power Eng. China 1 (4) (2007) 413-419. doi:10.1007/s11708-007-0060-8.

[51] M. Antal, S. G. Allen, X. Dai, B. Shimizu, M. S. Tam, M. Grønli, Attainment of the theoretical yield of carbon from biomass, Ind. Eng. Chem. Res. 39 (2000) 4024-. doi:10.1021/ie000511u. 


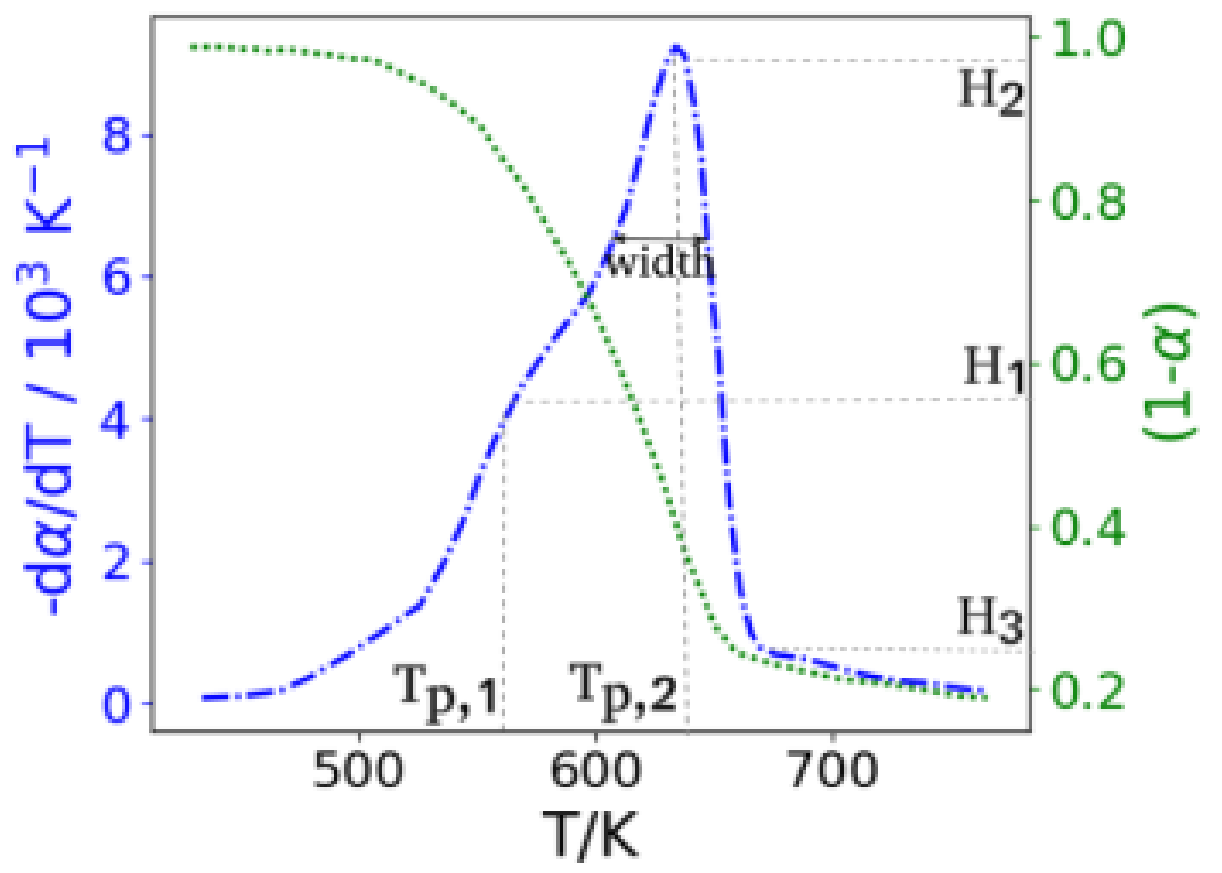

(a)

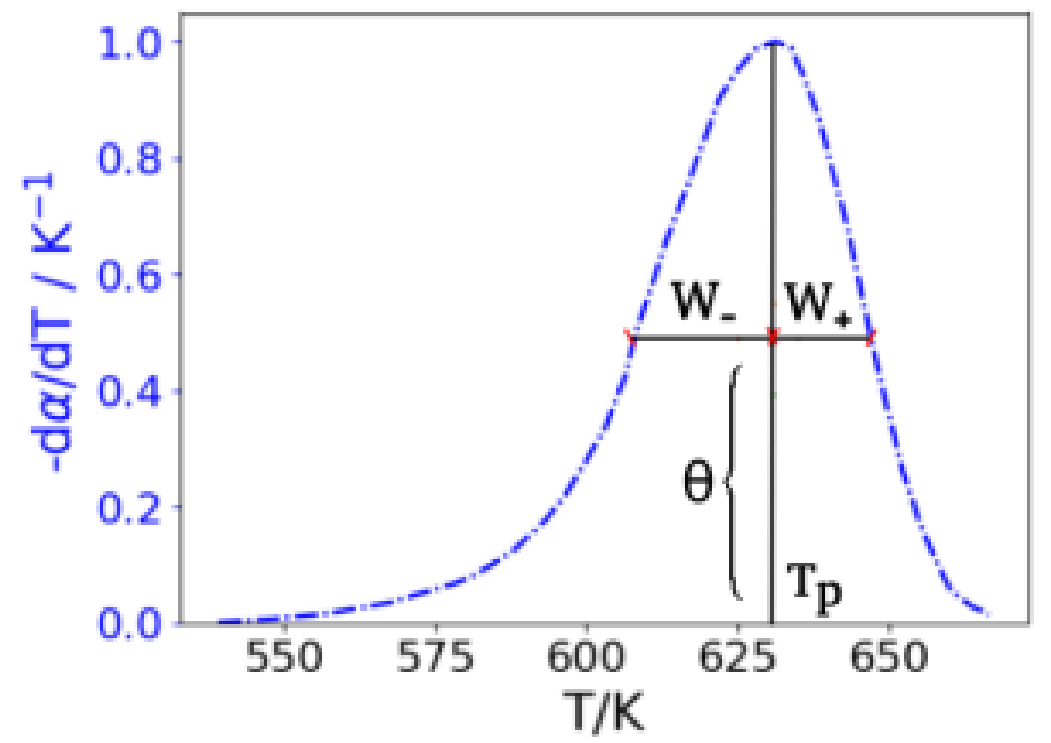

(b)

Figure 1: (a) Pyrolysis of a wood sample at $10 \mathrm{~K} \mathrm{~min}^{-1}$. The blue line is the mass loss curve, and the green line is the DTG curve [33]; $\mathrm{T}_{p}$ is the peak temperature, and $H$ is the peak height. (b) Pyrolysis of cellulose at $10 \mathrm{~K} \mathrm{~min}^{-1}$ [34]; $W$ is the width from the center to the side of the peak. 


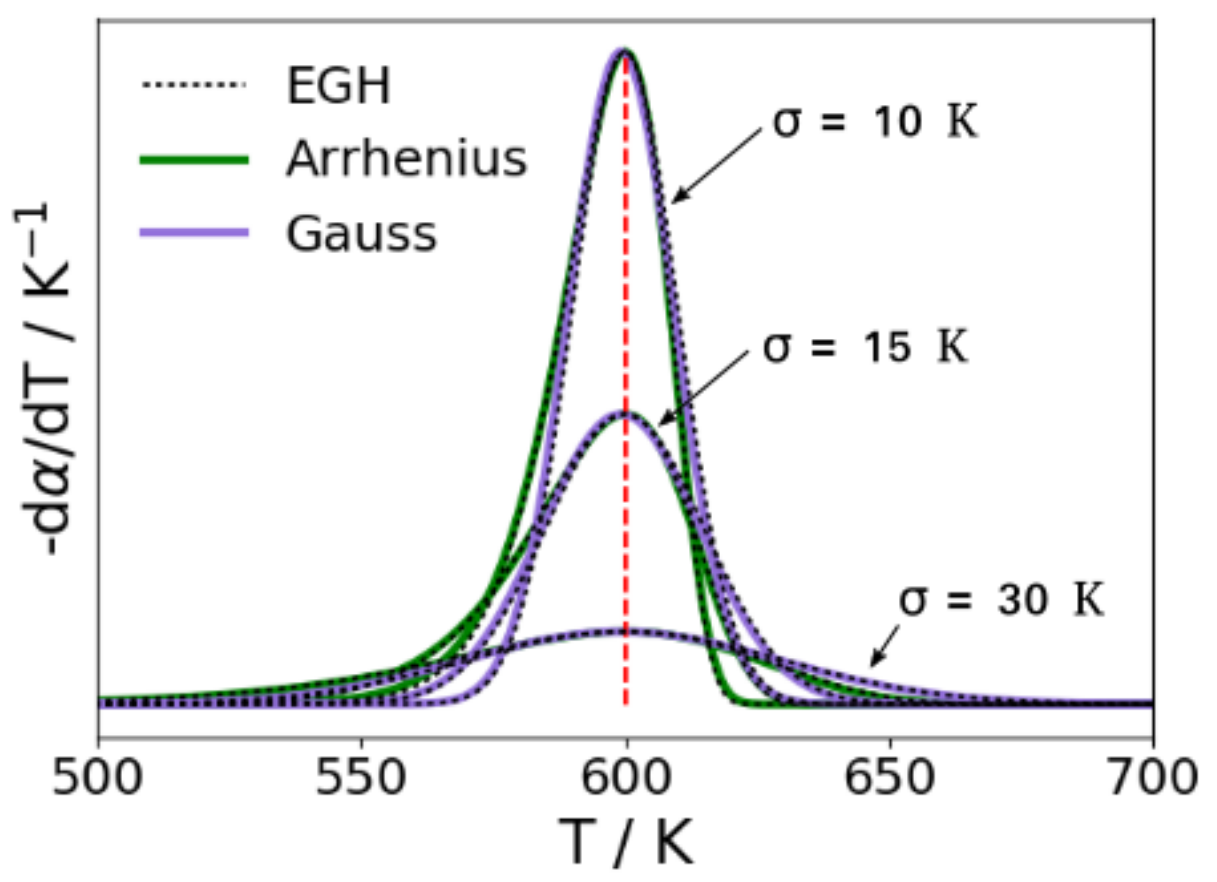

Figure 2: Comparison of peaks at $T_{p}=600 \mathrm{~K}$ and at different values of $\sigma$ for a Gaussian distribution, an Arrhenius first-order model with the calculated kinetic parameters for $\sigma=10 \mathrm{~K}$ of $E=300.0 \mathrm{~kJ} \mathrm{~mol}^{-1}, A=9.5 \times 10^{23} \mathrm{~s}^{-1}$ and RMS $=0.06 \%$, for $\sigma=15 \mathrm{~K}$ of $E=200.0 \mathrm{~kJ} \mathrm{~mol}^{-1}$, $A=1.3 \times 10^{15} \mathrm{~s}^{-1}$ and RMS $=0.03 \%$, for $\sigma=30 \mathrm{~K}$ of $E=100.0 \mathrm{~kJ} \mathrm{~mol}^{-1}, A=1.3 \times 10^{6} \mathrm{~s}^{-1}$ and RMS $=0.0 \%$, and the EGH model corresponding to the Gaussian distribution with $\tau=0$ and corresponding to the Arrhenius first-order model with $\tau=-6 \mathrm{~K}$ and RMS $=0.01 \%$ for $\sigma=10 \mathrm{~K}$, $\tau=-8.6 \mathrm{~K}$ and $\mathrm{RMS}=0.01 \%$ for $\sigma=15 \mathrm{~K}$, and $\tau=-14.6 \mathrm{~K}$ and $\mathrm{RMS}=0.01 \%$ for $\sigma=30 \mathrm{~K}$. 


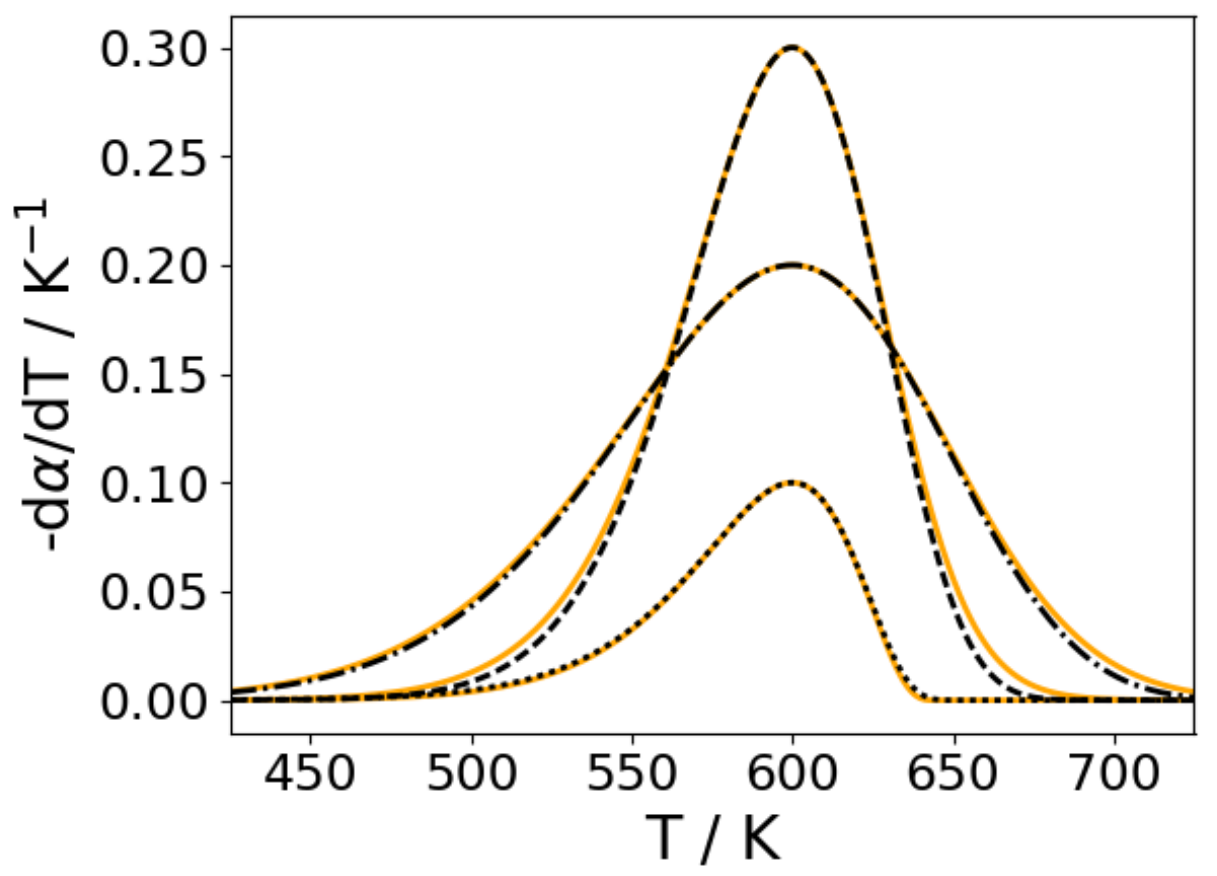

Figure 3: Peaks comparison of EGH model and $n$ th-order Arrhenius at $20 \mathrm{~K} \mathrm{~min}^{-1}$ and $T_{p}=600 \mathrm{~K}$. Discontinuous line: EGH model, dashed-line corresponds to $H_{p}=0.3 \mathrm{~K}^{-1}, \sigma=30 \mathrm{~K}$ and $\tau=-10 \mathrm{~K}$, dash-dot line $H_{p}=0.2 \mathrm{~K}^{-1}, \sigma=50 \mathrm{~K}$ and $\tau=-15 \mathrm{~K}$, and dotted-line $H_{p}=0.1 \mathrm{~K}^{-1}$ with, $\sigma=25 \mathrm{~K}$ and $\tau=-20 \mathrm{~K}$. Solid line: calculated Arrhenius $n$ th-order, the calculated parameters corresponding to the EGH curve in dashed-line are $E=103.3 \mathrm{~kJ} \mathrm{~mol}^{-1}, A=1.1 \times 10^{7} \mathrm{~s}^{-1}, n=1.2$ and a RMS $=6.0 \%$, for the dash-dot line $E=57.9 \mathrm{~kJ} \mathrm{~mol}^{-1}, A=6.9 \times 10^{2} \mathrm{~s}^{-1}, n=1.1$ and a RMS $=5.1 \%$, and for the dotted line $E=100.9 \mathrm{~kJ} \mathrm{~mol}^{-1}, A=7.0 \times 10^{6} \mathrm{~s}^{-1}, n=0.8$ and a RMS $=0.0 \%$. 


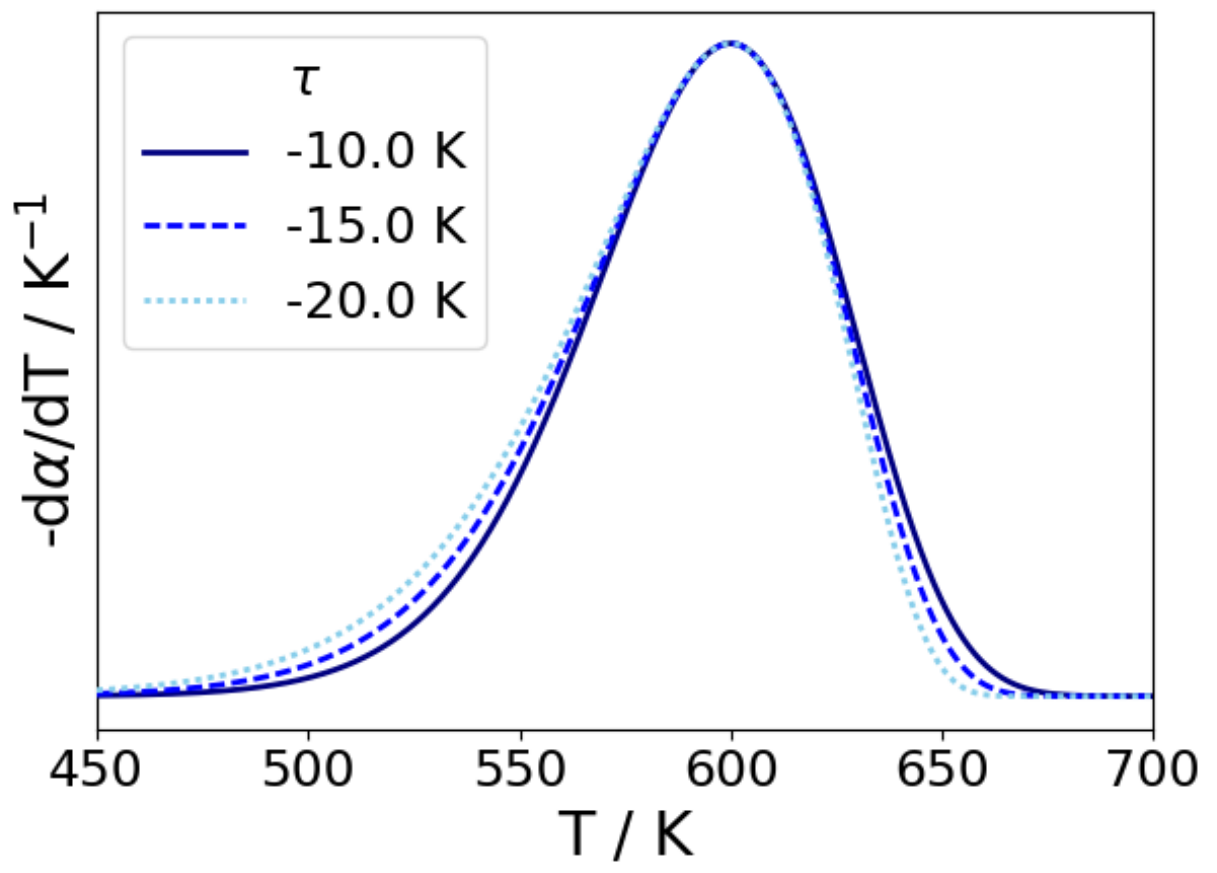

Figure 4: EGH model peaks comparison at $20 \mathrm{~K} \mathrm{~min}^{-1}, T_{p}=600 \mathrm{~K}$ and $\sigma=30 \mathrm{~K}$ for different values of $\tau$. 


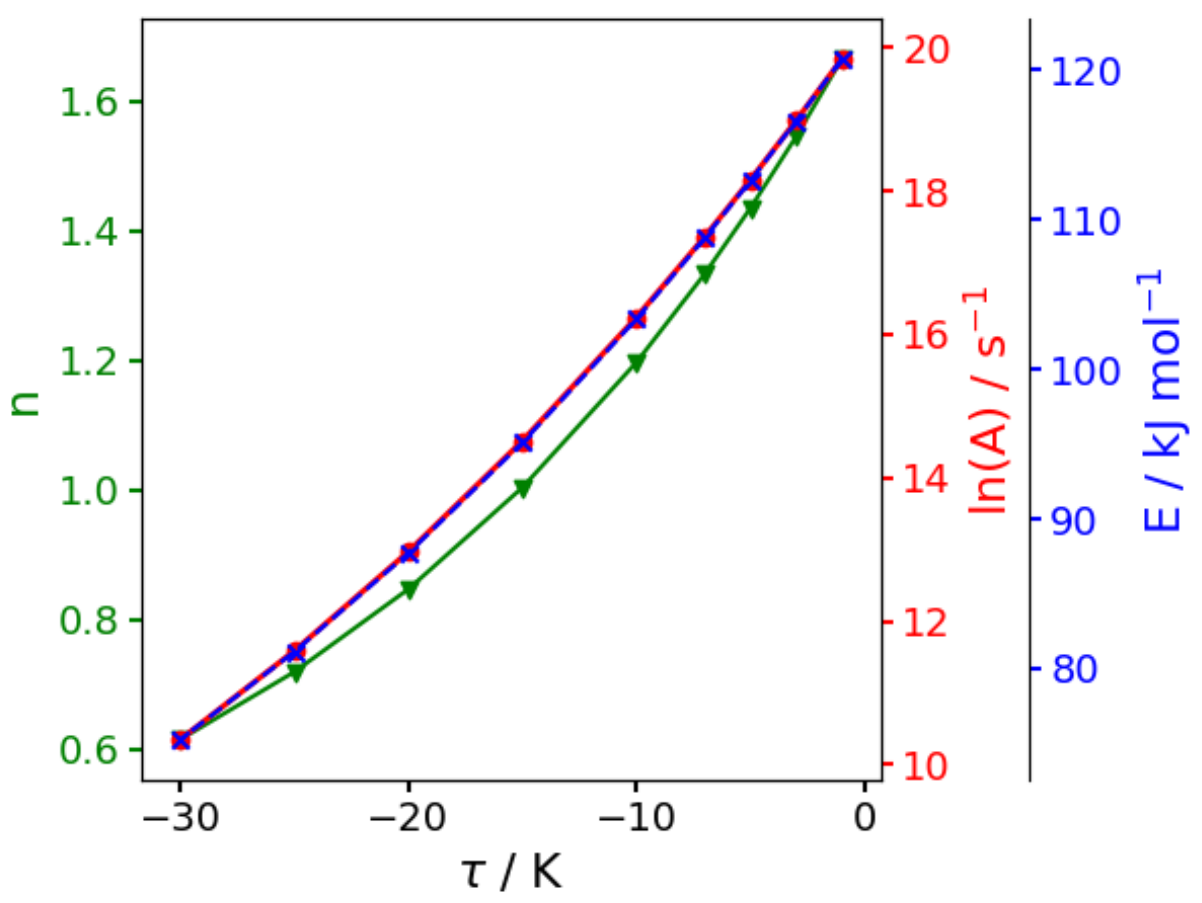

Figure 5: Corresponding values for the kinetic parameters of an $n$ th-order Arrhenius model from the EGH model, for a heating rate of $20 \mathrm{~K} \mathrm{~min}^{-1}$, $T_{p}=600 \mathrm{~K}$ and $\sigma=30 \mathrm{~K}$, with different values of $\tau$. 


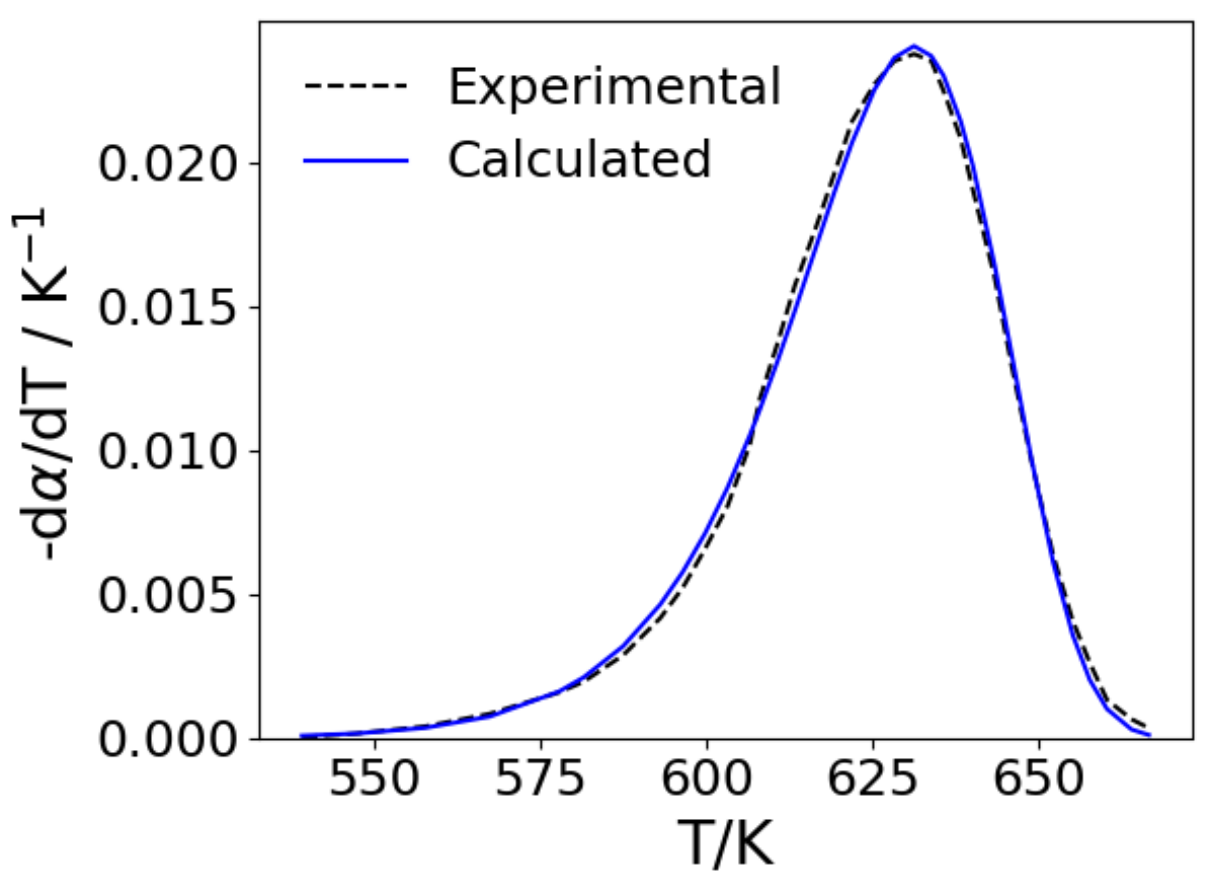

Figure 6: Calculated and experimental DTG curve of cellulose pyrolysis at $10 \mathrm{~K} \mathrm{~min}^{-1}$ [34].

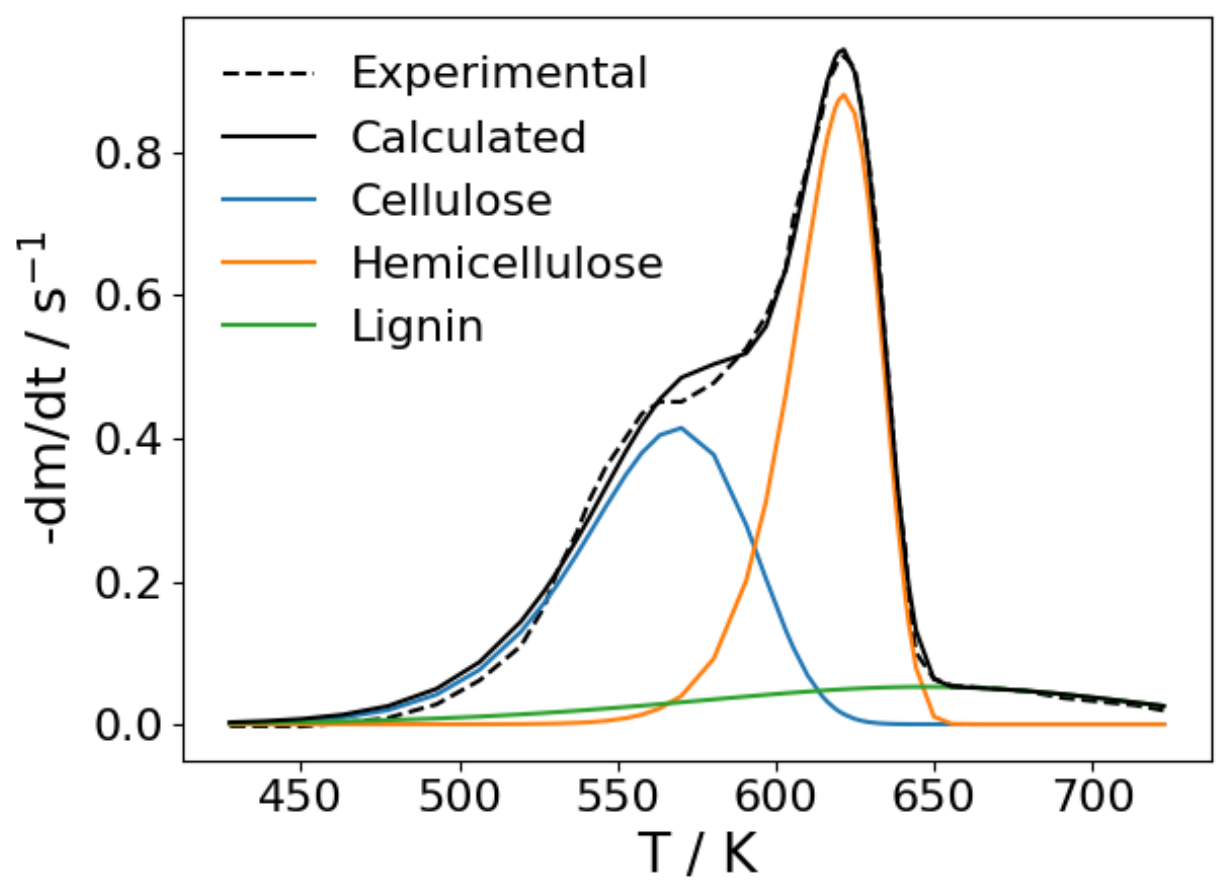

Figure 7: Deconvolution of experimental data of beech wood pyrolysis at $5 \mathrm{~K} \mathrm{~min}^{-1}$ [15]. 


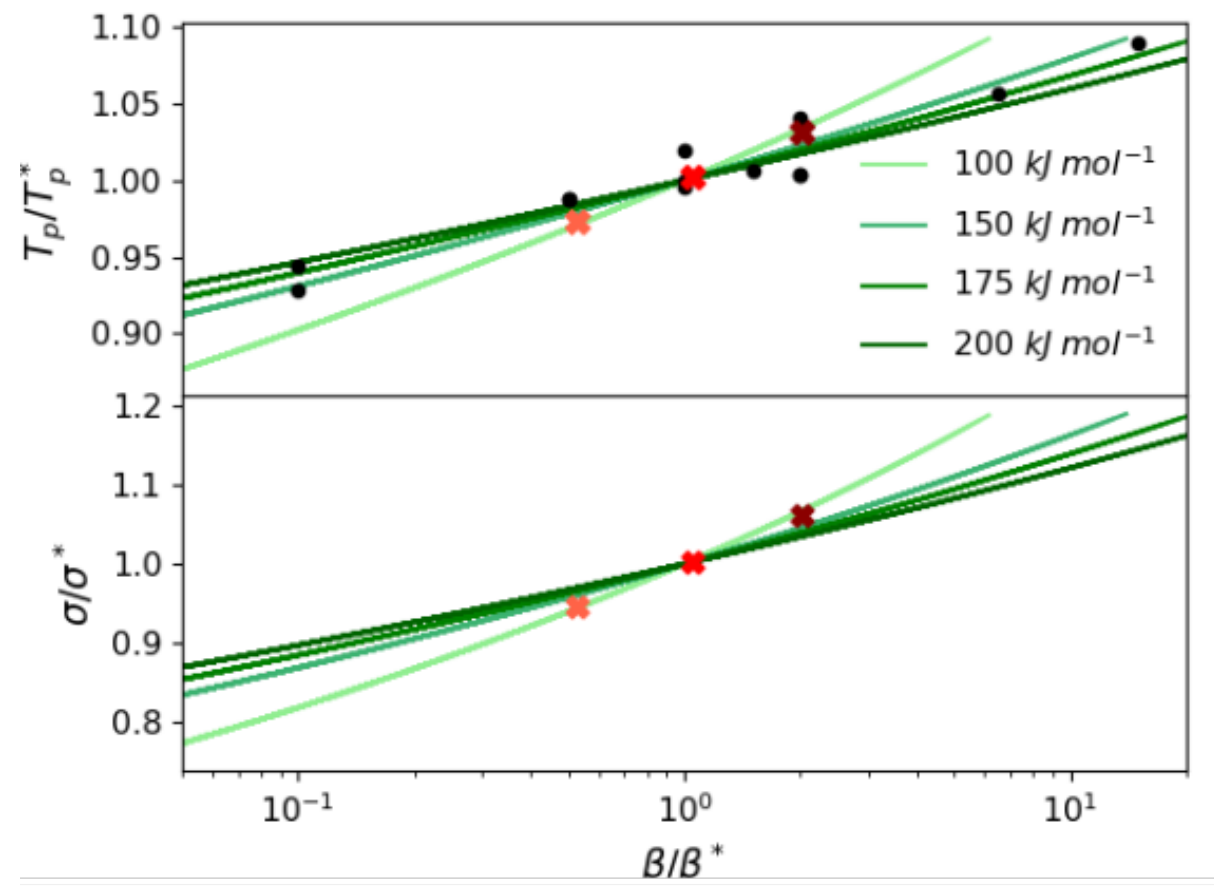

(a)

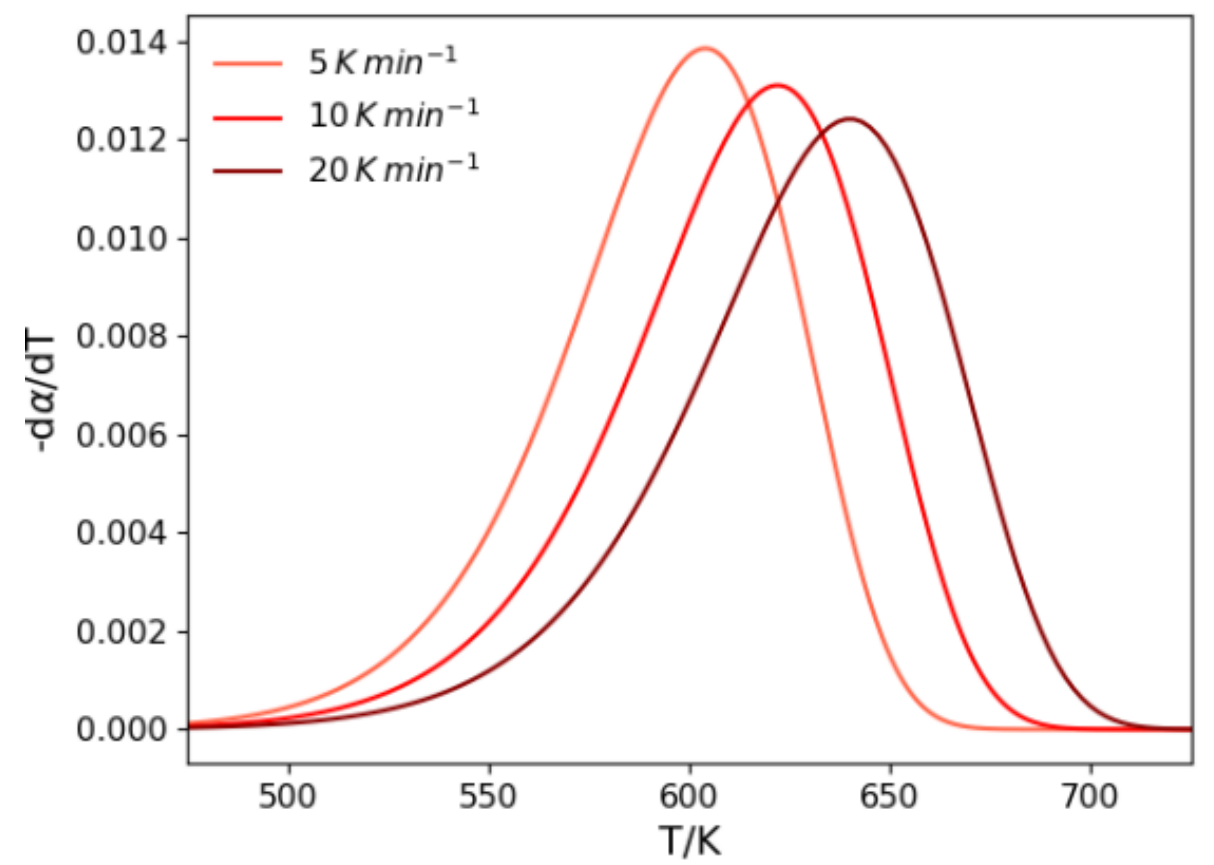

(b)

Figure 8: (a) Different activation energy curves for cellulose, where the black dots are experimental data of peak temperature versus heating rate $[34,46,47,48,49,19,21,20,50]$, and the red crosses are calculated points from b. (b) Calculated DTG curves for cellulose, derived for E=100 $\mathrm{kJ} / \mathrm{mol}$. 


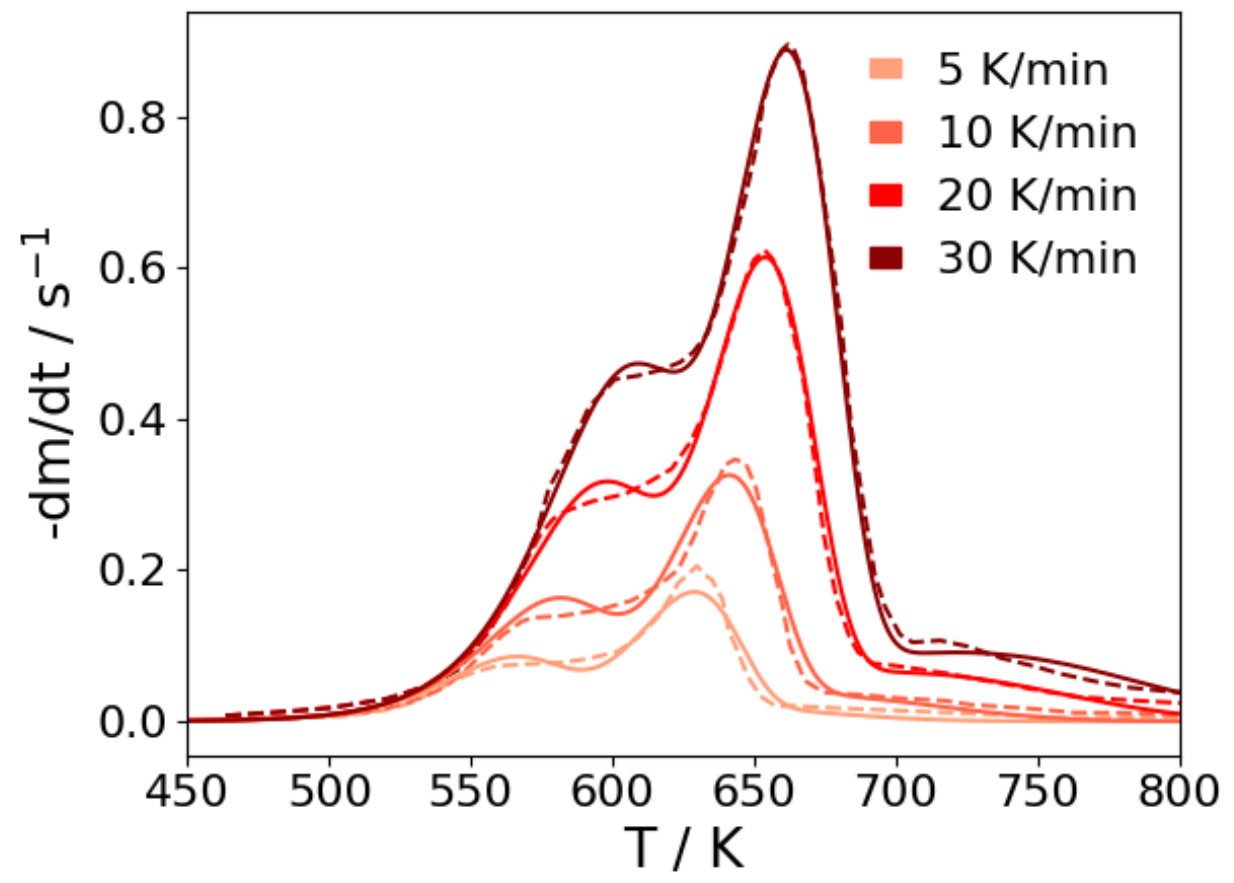

Figure 9: Simultaneous evaluation of experimental data from macadamia nut shell pyrolysis at different heating rates [45]; dashed line: experimental, solid line: calculated. 